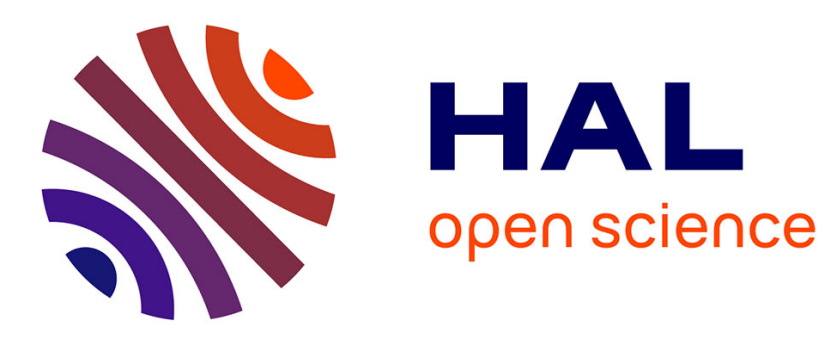

\title{
Influence of water spraying on an oscillating channel
}

\author{
Anne Bouvet, Xavier Pelorson, Annemie van Hirtum
}

\section{To cite this version:}

Anne Bouvet, Xavier Pelorson, Annemie van Hirtum. Influence of water spraying on an oscillating channel. Journal of Fluids and Structures, 2020, 93, pp.102840. 10.1016/j.jfluidstructs.2019.102840 . hal-02415110

\section{HAL Id: hal-02415110 https://hal.univ-grenoble-alpes.fr/hal-02415110}

Submitted on 28 May 2020

HAL is a multi-disciplinary open access archive for the deposit and dissemination of scientific research documents, whether they are published or not. The documents may come from teaching and research institutions in France or abroad, or from public or private research centers.
L'archive ouverte pluridisciplinaire HAL, est destinée au dépôt et à la diffusion de documents scientifiques de niveau recherche, publiés ou non, émanant des établissements d'enseignement et de recherche français ou étrangers, des laboratoires publics ou privés. 


\title{
Influence of water spraying on an oscillating channel
}

\author{
Anne Bouvet, Xavier Pelorson, Annemie Van Hirtum ${ }^{1}$ \\ LEGI, UMR CNRS 5519, Grenoble Alpes University, France
}

\begin{abstract}
The influence of surface hydration on the fluid-structure instability underlying vocal folds auto-oscillation during voiced speech sound production is an open research question. In this work the influence of homogeneous water spraying on an oscillating channel is investigated experimentally using several vocal folds replicas. Changes to glottal flow features are systematically quantified for a rigid replica with forced oscillation. Changes to auto-oscillation features are systematically quantified by analysing the pressure measured upstream from deformable replicas. During auto-oscillation it is observed for increasing water volume that the first harmonic frequency decreases, its amplitude increases, cycle-to-cycle as well as overall fluctuations increase and the closing-opening asymmetry changes. Nevertheless, the magnitude of these effects differs between deformable replicas so that further systematic investigation is needed to quantify observations as well as to explore underlying mechanisms. Flow tendencies observed on all replicas support that water spraying affects the glottal flow rather then structural properties. This is an important finding for future modeling of the effect of water spraying on the fluid-structure interaction.

Keywords: Two-phase flow, Vocal fold surface hydration, Mechanical vocal folds replicas, fluid-structure interaction
\end{abstract}

\footnotetext{
${ }^{1}$ annemie.vanhirtum@univ-grenoble-alpes.fr
} 


\section{Introduction}

The surface of human vocal folds (VF), illustrated in Fig. 1, is hydrated with a liquid layer $[1,2]$. It is established from observations on human speakers and on cadaver larynges that good surface hydration may diminish phonotraumatic VF

5 lesions [1,3] and depends on a combination of environmental factors [4], vocal effort $[5,6]$, life style $[7,8]$ and general health state [9]. Artificial saliva sprays are developed to remedy VF surface hydration $[10,11,4]$ so that liquid sprays and nebulisation techniques are common in (re-)hydration studies focusing on voice properties $[12,11,13]$. One of the physical consequences attributed to surface 10 hydration is to reduce the auto-oscillation onset pressure, i.e. the minimum subglottic pressure required to sustain VF auto-oscillation or phonation [14, $3,15,16]$. Other voice auto-oscillation properties are sensitive to hydration such as oscillation frequency $f_{0}$, closing quotient, speed quotient and cycle-tocycle perturbations jitter and shimmer $[12,2,13,17]$. Nevertheless, different tendencies of these features are described in literature stressing the need for physical studies. So far, few physical studies of the VF instability reported in literature deal with the effect of surface hydration.

From a physical point of view, VF auto-oscillation during voiced speech sound production is due to a fluid-structure (FS) instability in the larynx fol-

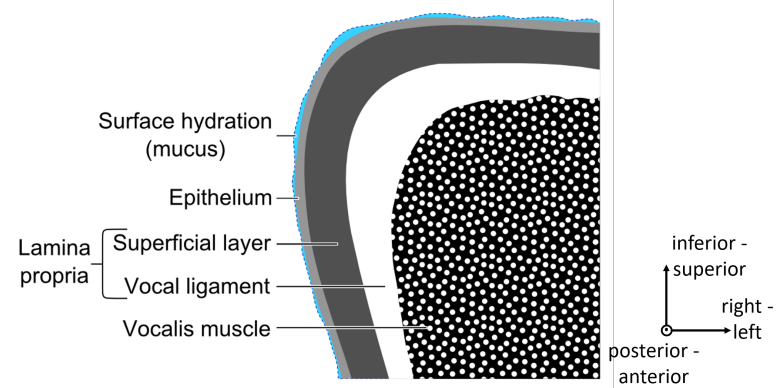

Figure 1: Illustration of layered structure of human VF with surface hydration in medio-frontal plane. Based on [18]. 
lowing the interaction of airflow coming from the lungs and the enveloping VF tissues. This FS interaction (FSI) mechanism can be represented as a deformable VF with one mechanical degree of freedom acted on by the flow while assuming a constant time delay associated with the time needed for the deformation wave to travel along the VF surface $[19,20,21,22]$. The variation of transverse area $A(t)$ associated with each streamwise mass position is then modeled considering the equation of transverse motion (left hand side) which is excited from its transverse rest position $A_{0}$ by an acting fluid force $F_{A}$ (right hand side) so that

$$
m \ddot{A}(t)+r \dot{A}(t)+k\left(A(t)-A_{0}\right) \approx F_{A}(t),
$$

with vibrating mass $m$, damping $r$ and stiffness $k$. Recently, it was shown using a motion driven rigid VF replica that the glottal pressure drop is affected after water spraying [23]. The mean flow was affected by the air-water mixture during the opening and closing phase of the glottal cycle in different ways leading to an altered opening-closing asymmetry. Therefore, fluid force $F_{A}(t)$ in Eq. (1) is likely to depend on the sprayed water volume which in turn affects the fluidstructure interaction and hence the VF's auto-oscillation.

In this work, flow features and their perturbation often associated with voice quality are systematically quantified from experiments mimicking water spraying on oscillating vocal folds firstly using a motion driven rigid VF replica and secondly using deformable VF replicas. This way hydration after usage of an artificial saliva spray is mimicked. Experiments with the motion driven rigid VF replicas allow to systematically quantify flow features within the glottis as the pressure within the constriction is analysed. Quantification is of interest as no such features were quantified in [23] and glottal flow features are directly 35 related to $F_{A}(t)$ in Eq. (1). Experiments with deformable mechanical VF replicas allow to systematically quantify flow features during auto-oscillation as the upstream pressure is analyzed. Tendencies observed on the quantified features for the glottal flow (rigid VF replica) and during auto-oscillation (deformable VF replicas) are considered in order to assess that in terms of Eq. (1) water 
40 spraying affects the flow expressed by $F_{A}(t)$ rather then mechanical properties $m, r$ and $k$. This is of interest for future modelling studies of the fluid-structure interaction with surface hydration due to liquid spraying. In the following, vocal fold replicas are described in Section 2, the use of water as a spraying liquid is motivated in Section 3 and the experimental procedures are detailed

45 in Section 4. Experimental results are presented in Section 6 and discussed in Section 7. The conclusion is formulated in Section 8.

\section{Dynamic mechanical VF replicas}

Different dynamic mechanical VF replicas are assessed allowing to experiin Eq. (1): motion driven rigid (duralium, neutral water wet wettability) MDR in Section 2.1 [24, 23], deformable silicone casts (M5, MRI and EPI) in Section $2.2[25,26,27,28,29,30,31]$ and deformable pressurized latex tube PLT in Section $2.3[32,20,22]$. Both deformable VF replica types are of interest ity) VF replicas allow to represent the multi-layer structure of human VF's with different degree of complexity whereas pressurized latex (neutral water wet wettability) tubes allow to mimic the variation of human VF's elasticity in a systematic way. The motion driven rigid MDR replica allows to observe the 60 glottal flow which can not be achieved for deformable VF replicas.

Water is not absorbed by any of the mechanical VF replicas with smooth surface, either rigid or deformable. Consequently, their structural properties $(m, r$ and $k$ in Eq. (1)) are not affected by absorption when in contact with water.

65

Each of the assessed dynamic mechanical VF replicas (posterior-anterior width $w$ and inferior-superior height $L_{z}$ ) is positioned so that glottal-like area $A_{c}$, i.e. the spacing between both VFs, is situated in a transverse horizontal plane in such a way that the interior edges of the right and left VFs are paral- 


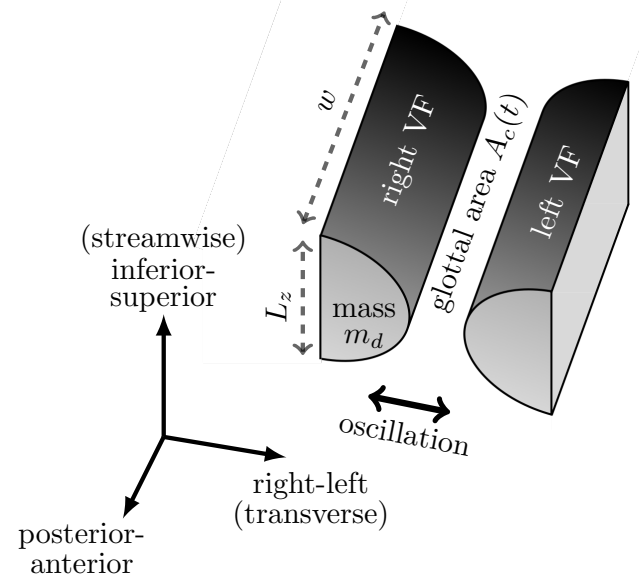

Figure 2: Oscillating VF replicas: width $w$, height $L_{z}$, glottal area $A_{c}(t)$ and mass $m_{d}$.

lel to the posterior-anterior axis as depicted in Fig. 2. The glottal area $A_{c}(t)$ is varied from its initial value $A_{0}$ during VFs oscillation along the transverse direction (right-left axis). Auto-oscillation occurs using deformable VF replicas (M5, MRI, EPI and PLT) due to a fluid-structure interaction with the airflow passing through the glottal area. Forced oscillation is imposed using the rigid VF replica (MDR). An overview of VF properties for used replicas and for a male adult is given in Table 1: $L_{z}, w, A_{c, \max }, A_{0}$ and $m_{d}$ with maximum glottal area $A_{c, \max }$ so that $A_{c}(t) \leq A_{c, \max }$ and initial glottal area $A_{c}(t=0)=A_{0}$.

\subsection{Motion driven rigid: $M D R$}

The motion driven rigid (MDR) VF replica depicted in Fig. 3 consists of two rigid parallel half cylinders (radius $10 \mathrm{~mm}, L_{z}=20 \mathrm{~mm}, w=30 \mathrm{~mm}$ ), one of which is forced into motion by an eccentric motor [36, 24]. Sinusoidal movement of the rectangular gap between both half cylinders is imposed with forcing frequency $f_{c} \in\{1,6,10\} \mathrm{Hz}$ and constant forcing amplitude. The imposed time-varying minimum spacing $h_{c}(t)\left(0 \leq h_{c} \leq 0.91 \mathrm{~mm}\right)$ is measured by means of an optical sensor (OPB700, accuracy $\pm 0.01 \mathrm{~mm}$ ), so that the time- 
Table 1: VF replicas and human: dimensions and mass.

\begin{tabular}{l|ccccc}
\hline & $L_{z}^{\star}$ & $w^{\dagger}$ & $A_{c, \text { max }}{ }^{\ddagger}$ & $A_{0} \vee$ & $m_{d}{ }^{\square}$ \\
\hline \hline MDR & 20.0 & 30.0 & 60 & - & - \\
\hline M5 & 10.7 & 17.0 & 85 & $<10$ & 2.3 \\
MRI & 10.0 & 18.0 & 85 & $<10$ & 1.8 \\
EPI & 10.2 & 17.0 & 85 & $<10$ & 1.9 \\
PLT & 12.0 & 25.0 & 100 & $18-74$ & 2.1 \\
\hline adult & $4-8$ & $15-25$ & 50 & $<20$ & - \\
\hline
\end{tabular}

^ $L_{z}$ : inferior-superior VF length, $[\mathrm{mm}]$

$\dagger w$ : posterior-anterior VF width, $[\mathrm{mm}]$

$\ddagger$ upper limit for glottal area as $\max \left(A_{c}(t)\right) \leq A_{c, \max },\left[\mathrm{mm}^{2}\right]$

$\vee A_{0}=A_{c}(t=0)$ : initial glottal area, $\left[\mathrm{mm}^{2}\right]$

$\square m_{d}$ : VF mass for $V_{L}=0 \mathrm{~mL}$, [g]

$\triangleleft$ male adult $[33,34,35]$

varying glottal area yields $A_{c}(t)=h_{c}(t) \cdot w$ and $A_{c, \max }=60 \mathrm{~mm}^{2}$ (Table 1). A pressure tap is foreseen in the fixed VF to measure constriction pressure $P_{c}(t)$ at minimum spacing.

\subsection{Deformable silicone casts: M5, MRI and EPI}

Three deformable silicone VF replicas - labeled M5, MRI and EPI - are obtained following the procedures detailed in $[25,26,30]$. Each VF consists of an overlay of two (M5), three (MRI) or four (EPI) molding layers (silicone thinner and two-part A\&B Ecoflex 03-00, Smooth-On, Inc., Easton, PA) in order to mimic the multi-layered human VF structure (Fig. 4). 


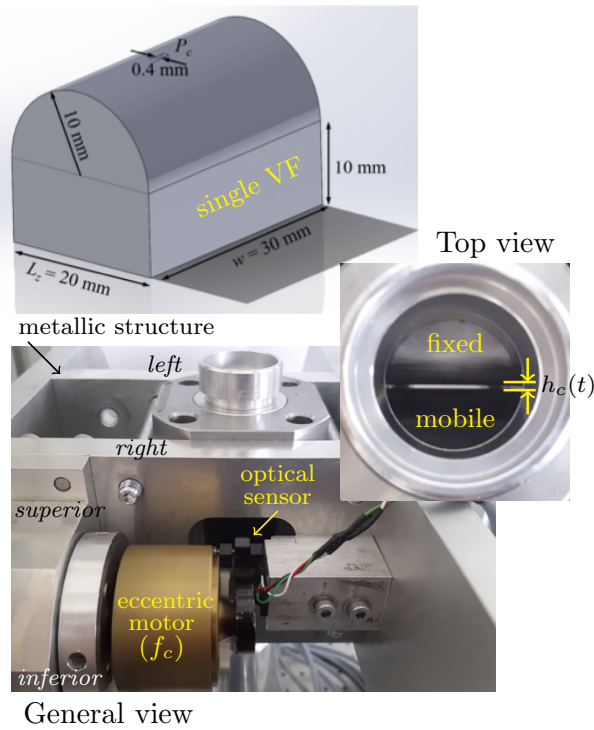

General view

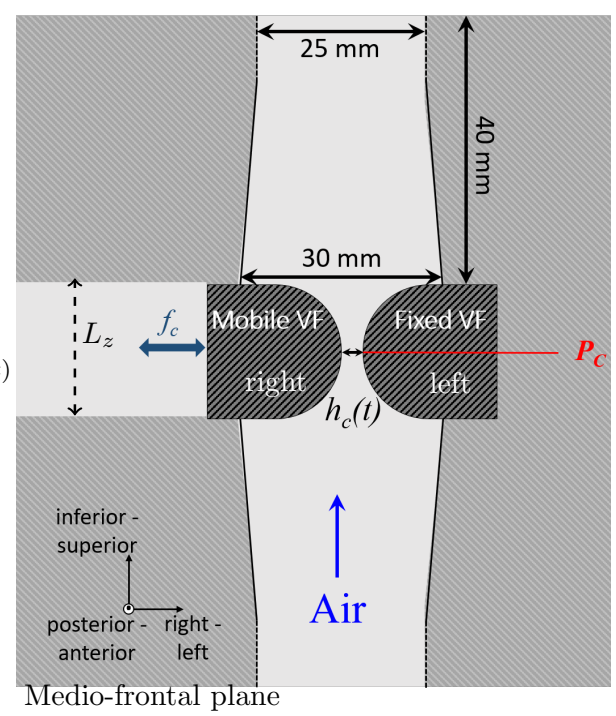

Medio-frontal plane

Figure 3: Motion driven rigid (MDR) VF replica.

\subsubsection{Different molding layers}

Each molding layer has a different ecoflex-to-silicone mixing ratio $\mathcal{M}$ so that from literature $[33,37,38,39,40,26]$ is seen (Table 2) that the order of magnitude of Young moduli $\mathcal{E}$ (tensile tests, INSTRON 3369 electro-mechanical press) is consistent with the thyroarytenoid (vocalis) muscle, the superficial layer of the lamina propria (Reinke's space) and epithelium layer whereas it is underestimated for the ligament (intermediate and deep layers of the lamina propria) since no tension is applied in the deep layer. Note that $\mathcal{E}$-values are consistent with the ones reported in literature [25]. Each VF is mounted on a backing layer (silicone thinner and two-part A\&B Dragon Skin FX Pro, Smooth-On, Inc., Easton, PA, ratio $\mathcal{M}$ 1:1, thickness $4 \mathrm{~mm}$ ) in order to attach the replica to its rigid support, i.e. region outside of the black frames in the top views of Fig. 4. 


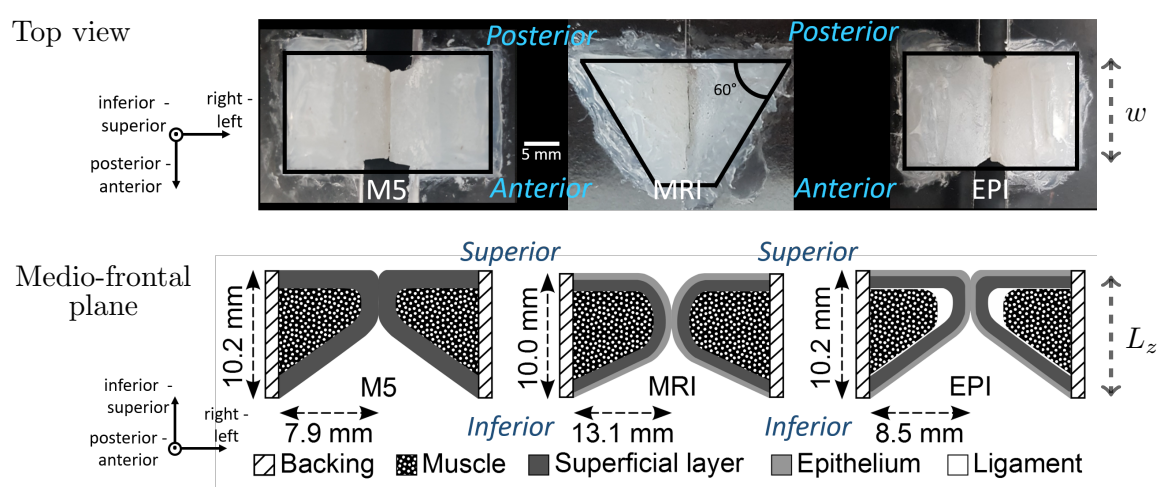

Figure 4: Deformable silicone VF replicas M5, MRI and EPI.

Dimensions and masses for all silicone VF replicas are summarized in Table 1: initial glottal area $A_{0}<10 \mathrm{~mm}^{2}, A_{c, \max }=85 \mathrm{~mm}^{2}$, inferior-superior 
Table 2: Layer properties in human and in silicone VF replicas $\left(\mathrm{M}^{\star}, \mathrm{MRI}^{\dagger}, \mathrm{EPI}^{\ddagger}\right)[33,37$, $38,39,40,26]$ : Young modulus $\mathcal{E}$, ecoflex-to-silicone ratio $\mathcal{M}$, layer thickness $l_{d}$.

\begin{tabular}{l||cc|ccc}
\hline \multirow{2}{*}{ Layer } & \multicolumn{2}{|c|}{ Male adult } & \multicolumn{3}{|c}{ VF replica } \\
\hline Muscle $^{E S}$ & $8-29$ & 6.0 & $10.4^{\star} 4.9^{\dagger} 21.9^{\ddagger}$ & $1: 2$ & $6.4^{\star} 10.0^{\dagger} 6.4^{\ddagger}$ \\
Superficial $^{E S}$ & $2-9$ & 0.6 & $4.9^{\star} 0.2^{\dagger, \ddagger}$ & $1: 4$ & $1.5^{\star} 3.0^{\dagger} 1.0^{\ddagger}$ \\
Ligament $^{E S}$ & $10-45$ & 0.8 & $4.9^{\ddagger}$ & $1: 8$ & $1.0^{\ddagger}$ \\
Epithelium $^{D S}$ & $40-60$ & 0.1 & $52^{\dagger, \ddagger}$ & $1: 1$ & $0.1^{\dagger, \ddagger}$ \\
\hline
\end{tabular}

* M5: muscle and superficial

$\dagger$ MRI: muscle, superficial and epithelium

‡ EPI: muscle, superficial, ligament and epithelium

$E S$ Ecoflex/Silicone mixture

${ }^{D S}$ Dragonskin/Silicone mixture

length $L_{z} \approx 10 \mathrm{~mm}$, posterior-anterior width $w \approx 17 \mathrm{~mm}$ and silicone $\mathrm{VF}$ masses $m_{d}<2.5 \mathrm{~g}$.

\subsection{Deformable pressurized latex tube: PLT}

The deformable pressurized latex tube (PLT) VF replica $\left(L_{z}=12 \mathrm{~mm}\right.$, $w=25 \mathrm{~mm}$, mass $m_{d}=2.1 \mathrm{~g}$ in Table 1) is illustrated in Fig. 5 and detailed in $[22,42]$. Each VF consists of a latex tube (diameter $11 \mathrm{~mm}$, thickness $0.2 \mathrm{~mm}$ ) which is pressurized $\left(P_{P L T}\right)$ by filling it with distilled water which is connected to a water column whose height can be changed. Therefore, the elasticity of each VF can be varied by changing the internal pressure $P_{P L T}$ of each latex tube. Pressurised VFs are mounted in a metallic holder in a way that the initial position of each VF with respect to the centerline of the attached downstream and upstream tube, and hence the spacing between both VF's, can be varied using four micrometer screws (Mitutoyo 153-101, accuracy $0.01 \mathrm{~mm}$, range $5 \mathrm{~mm}$ ).

It is noted that initial glottal area $A_{0}$ is the combined result of imposed inter- 


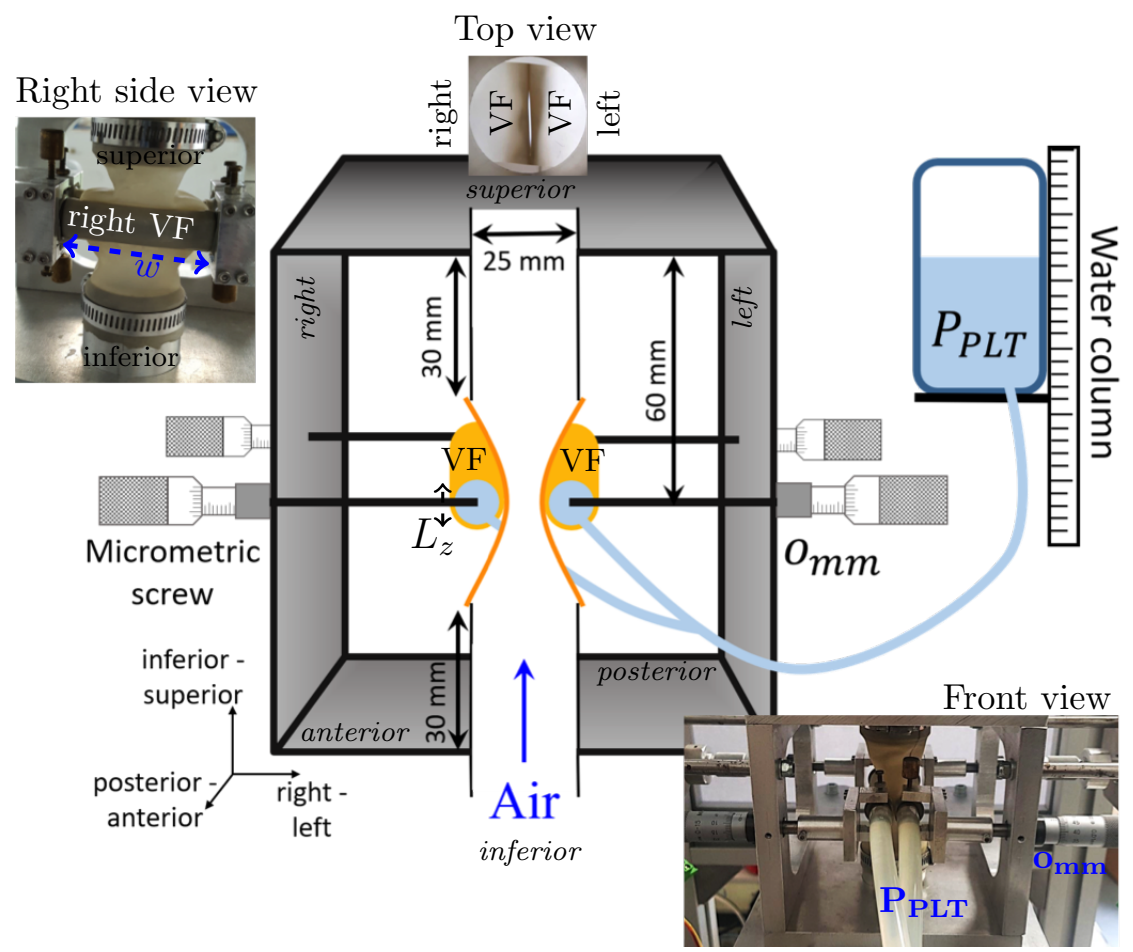

Figure 5: Deformable pressurized latex tube (PLT) VF replica and conditions $\left(P_{P L T}, o_{m m}\right)$.

nal pressure $P_{P L T}$ and the position of micrometer screws $o_{m m}$. For brevity, PLT conditions are denoted as $\left(P_{P L T}, o_{m m}\right)$. Concretely, 5 different PLT VF replica conditions are considered - indicated as $(2300,0),(2800,0),(3300,0),(2800,1)$ and $(2800,2)$ - so that either $o_{m m}$ or $P_{P L T}$ deflects from condition $(2800,0)$. The initial area $A_{0}$ ranges from $18 \mathrm{~mm}^{2}$ up to $74 \mathrm{~mm}^{2}$ and $A_{c, \max }=100 \mathrm{~mm}^{2}$ as indicated in Table 1.

\section{Hydration liquid: water}

Distilled water (density $\rho_{L}=998 \mathrm{~kg} \cdot \mathrm{m}^{-3}$, dynamic viscosity coefficient $\mu_{L}=1.0 \times 10^{-3} \mathrm{~Pa} \cdot \mathrm{s}$, temperature $22 \pm 2{ }^{\circ} \mathrm{C}$ ) is used as hydration liquid fluid. Water has well known properties so that it is a good reference for future studies 
Compared to airway mucus $[43,44]$ and saliva $[10,45]$, water has similar density, but a reduced (up to 2000-fold for airway mucus of healthy subjects) dynamic viscosity. Despite the difference in visco-elastic properties and the lack of salinity and of an elastic structural matrix, bidirectional water fluxes through the epithelium play an important role in regulating VF surface mucus ( $\leq 0.9 \mathrm{~mm}$ ) hydration since it consists of a superficial cover with high viscosity and thin $(\approx 15 \mu \mathrm{m})$ underlying aqueous layer with low viscosity [46, 47, 48, 2]. Therefore, water is an important component of VF surface liquid often used in (de-)hydration studies on human subjects $[2,11,17]$ and on cadaver larynges $[2,17,12]$. Nebulization of either sterile (hypotonic) water or physiological saline solutions is reported to result in a non-statistically significant difference as a remedy for laryngeal desiccation treatment [11]. It is important to note that natural human saliva and mucus are non-Newtonian fluids [10, 45, 43, 44, 49] whereas water is a Newtonian fluid. Nevertheless, some artificial saliva sprays contain a Newtonian fluid as well [10].

Typical liquid quantities for nebulization with artificial saliva sprays yield up to $4 \mathrm{~mL}$ [11]. Therefore, sprayed water volume $V_{L}$ is varied in the range

175 from 0 (dry, subscript $d$ ) up to $5 \mathrm{~mL}$ so that the total mass of sprayed water is $m_{V_{L}}=\rho_{L} \cdot V_{L}$.

\section{Experimental methods and operating conditions}

The overall setup with airflow supply is described in Section 4.1. Stable os180 cillations obtained using these setup without water spraying so that $V_{L}=0 \mathrm{~mL}$ are characterised for all replicas in Section 4.2. The experimental procedure used to assess oscillation after water spraying is detailed in Section 4.3. 


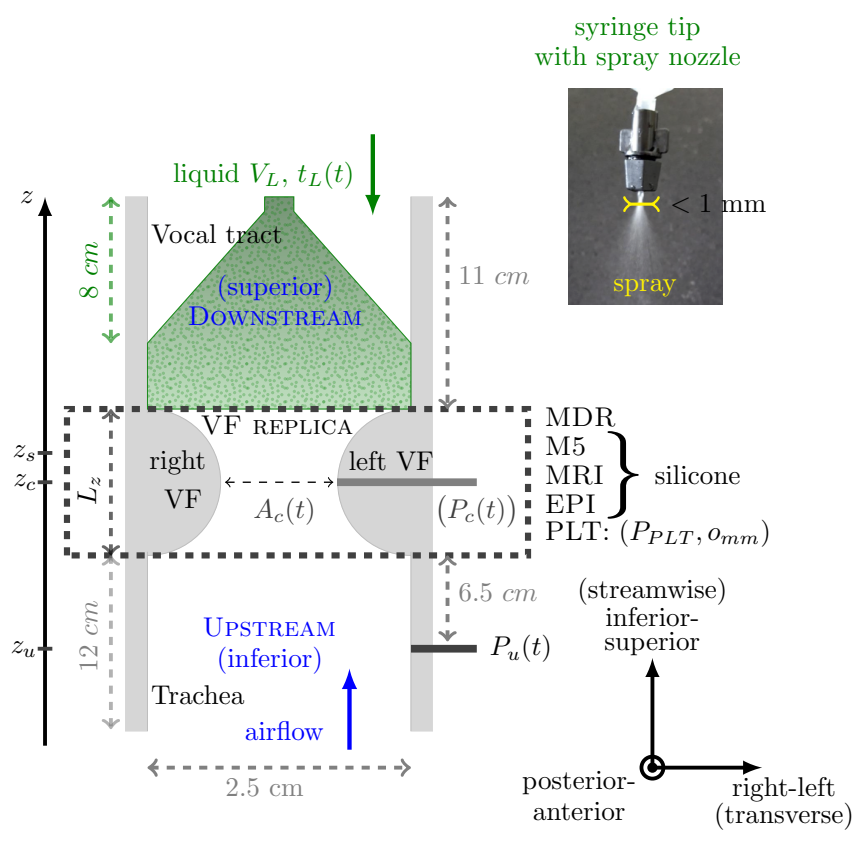

Figure 6: Schematic illustration of experimental setup with oscillating vocal folds replica in the medio-frontal plane indicating liquid injection time tag $t_{L}(t)$, sprayed liquid volume $V_{L}$, upstream pressure $P_{u}(t)$, glottal area $A_{c}(t)$. Assessed VF replicas: deformable (silicone and PLT) and forced motion (MDR with constriction pressure $P_{c}(t)$ ).

\subsection{Overall setup with airflow supply}

VF replicas, oriented as depicted in Fig. 2, are inserted in the overall experimental setup illustrated in Fig. 6. A rigid (smooth duralium, neutral water wet wettability) uniform circular channel with diameter $2.5 \mathrm{~cm}$ (constant area $490 \mathrm{~mm}^{2}$ ) is mounted vertically to the upstream (12 cm trachea inferior to VF) and downstream $(11 \mathrm{~cm}$ vocal tract superior to $\mathrm{VF})$ end of the VF replica. The downstream tube length is short compared to a typical vocal tract of a male adult $(\approx 18 \mathrm{~cm})$ in order to avoid acoustical coupling during VF oscillation. Note that from $A_{c, \max }$ values given in Table 1 (camera Mikrotron, EoSens Cube7, lens Tokina, AT-X100mmf/2.8 PRO D and calibration grid Edmuns Optics 58-607 [42]) follows that the area constriction ratio, i.e. the ratio between glottal area and constant area of the attached upstream and downstream tubes 
$\left(490 \mathrm{~mm}^{2}\right)$, is greater than $75 \%$ at all times for all VF replicas.

Continuous steady airflow (density $\rho_{G}=1.2 \mathrm{~kg} \cdot \mathrm{m}^{-3}$, dynamic viscosity $\mu_{G}=1.8 \times 10^{-5} \mathrm{~Pa} \cdot \mathrm{s}$, temperature $22 \pm 2^{\circ} \mathrm{C}$ ) is provided along the streamwise $z$-direction by a valve (Norgren, 11-818-987) controlled air supply. Air is delivered by an air compressor (Atlas Copco GA5 FF-300-8, GA15 FF-8) connected to an upstream pressure reservoir (volume $\geq 0.22 \mathrm{~m}^{3}$ ) filled with acoustic foam in order to avoid parasite acoustic resonances. A pressure transducer (Endevco $8507 \mathrm{C}-5$, accuracy $\pm 5 \mathrm{~Pa}$ ) is positioned in a pressure tap upstream of the $\mathrm{VF}$ replicas in order to measure upstream pressure $P_{u}(t)$. In the case of a motion driven rigid VF replica (MDR in Section 2.1) an additional pressure sensor is placed at the glottal spacing so that besides upstream pressure $P_{u}(t)$ constriction pressure $P_{c}(t)$ is measured as well, which allows to observe the glottal flow.

A stable oscillation is generated which is characterised by mean upstream pressure $\bar{P}_{u}$ and oscillation frequency $f_{0, d}$. An overestimation of volume flow rate $Q_{\max }$ is obtained as

$$
Q_{\max }=\sqrt{\frac{2 \bar{P}_{u}}{\rho_{G}}} A_{c, \max } \cdot c_{s}
$$

with glottal area upper limit $A_{c, \max }$ for each VF replica listed in Table 1. The separation constant $c_{s}=1.3$ defines a geometrical criterion for the time-varying position of flow detachment and jet formation $z_{s}$ (Fig. 6) as the lowest streamwise position with $z_{s}>z_{c}$ for which area $A_{z_{s}}(t) \geq c_{s} \cdot A_{c}(t)[36,24]$.

An upper limit for Reynolds number $R e$ is then given as

$$
R e_{\max }=\frac{\rho_{G} Q_{\max }}{\mu_{G} w},
$$

with $w$ as listed in Table 1 . Strouhal number $S r$ is estimated as

$$
S r=\frac{f_{0, d} L_{z}}{Q_{\max }} A_{c, \max } \cdot c_{s} .
$$


Table 3: Stable oscillation operating conditions before water spraying, $V_{L}=0 \mathrm{~mL}$.

\begin{tabular}{l|cccc}
\hline & $\bar{P}_{u}[\mathrm{~Pa}]$ & $f_{0, d}[\mathrm{~Hz}]$ & $R e_{\max }[-]$ & $S r[-]$ \\
\hline \hline M5 & 1750 & 97 & $<20 \times 10^{3}$ & $<2 \times 10^{-2}$ \\
MRI & 1500 & 114 & $<19 \times 10^{3}$ & $<2 \times 10^{-2}$ \\
EPI & 500 & 84 & $<11 \times 10^{3}$ & $<3 \times 10^{-2}$ \\
PLT & $250-500$ & $94-133$ & $<9 \times 10^{3}$ & $<5 \times 10^{-2}$ \\
\hline adult & $300-1000$ & $80-180$ & $\sim O\left(10^{3}\right)$ & $\sim O\left(10^{-2}\right)$ \\
\hline MDR & 1115 & $1-10$ & $\sim O\left(10^{3}\right)$ & $<0.5 \times 10^{-2}$ \\
\hline
\end{tabular}

Furthermore, a cycle-to-cycle maximum airflow velocity at the position of jet formation is estimated from the maximum upstream pressure within each oscillation cycle $P_{u, \max }$ as

$$
U_{P_{u, \max }} \approx \sqrt{\frac{2 P_{u, \max }}{\rho_{G}}} .
$$

Typical airflow velocities $U_{G}$ through the oscillating glottal-like constriction area vary from $0 \mathrm{~m} / \mathrm{s}$ up to $O\left(10^{1}\right) \mathrm{m} / \mathrm{s}[20,24]$.

Experiments to assess the effect of water spraying on an oscillating channel using this setup and airflow supply are further described in Section 4.3. All signals are acquired (PC/DAQ, National Instruments PCI-MIO-16XE-10) with sampling frequency $10 \mathrm{kHz}$ or a temporal accuracy of $0.1 \mathrm{~ms}$ which yields $\leq 1.5 \%$ of the period for a signal with oscillation frequency $\leq 150 \mathrm{~Hz}$.

\subsection{Stable oscillation operating conditions for $V_{L}=0 \mathrm{~mL}$}

Operating conditions are set so that stable oscillations occur before water spraying (subscript $d, V_{L}=0 \mathrm{~mL}$ ).

For the deformable VF replicas (PLT and silicone), mean upstream pressure $\bar{P}_{u}$ is set just above the onset threshold pressure $P_{o n}$, i.e. the minimum 


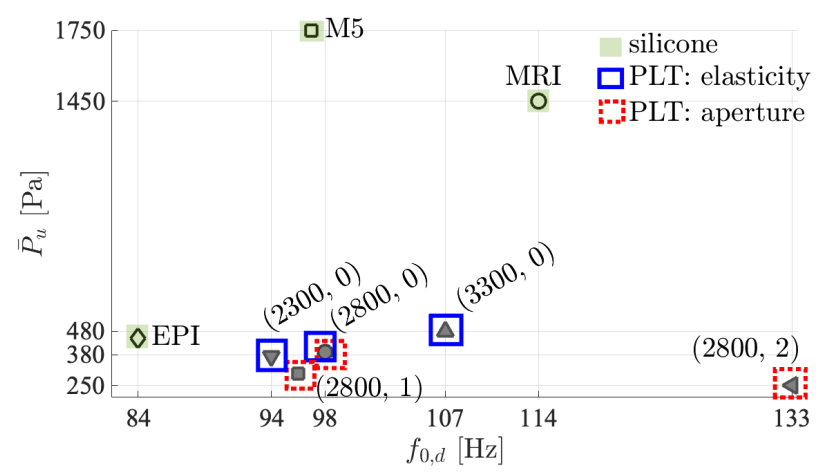

Figure 7: Deformable VF replicas in parameter space $\left(f_{0, d}, \bar{P}_{u}\right)$ for $V_{L}=0 \mathrm{~mL}$ : silicone VF replicas (M5, MRI, EPI: filled rectangles) and PLT VF replica conditions $\left(P_{P L T}, o_{m m}\right)$ with either $P_{P L T}$ (full line rectangles) or $o_{m m}$ (dashed rectangles) varied from reference condition $(2800,0)$.

upstream pressure required to sustain auto-oscillation. $P_{\text {on }}$ depends on the replica's elasticity so that it differs for each silicone VF replica and for each PLT condition $\left(P_{P L T}, o_{m m}\right)$. It follows that $\bar{P}_{u}$ differs for each replica as does oscillation frequency $f_{0, d}$. For the motion-driven replica $\bar{P}_{u}$ is set so that the Reynolds number approximates Reynolds numbers pertinent for human phonation and oscillation frequency $f_{c}$ is imposed so that $f_{0, d}=f_{c}$.

Table 3 summarises operating conditions for all VF replicas, deformable 235 (MRI, EPI, M5 and PLT) as well as motion-driven rigid (MDR), for mean upstream pressure $\bar{P}_{u}$, oscillation frequency $f_{0, d}$, Reynolds number $R e_{\max }$ (Eq. (3)) and Strouhal number $\operatorname{Sr}$ (Eq. (4)). As a reference, typical values observed for airflow through the glottis for a male adult speaker are also indicated in Table $3[33,34,35]$. For the motion driven rigid MDR VF replica $\bar{P}_{u}=1115 \mathrm{~Pa}$ 240 so that $R e_{\max }$ (Eq.(3)) is indeed of the same order of magnitude as observed for an adult male speaker whereas $\mathrm{Sr}$ (Eq. (4)) is lower. For all deformable VF replicas $S r$ is within the range for human phonation whereas $R e_{\max }$ is within this range for PLT and EPI and exceeds this range for M5 and MRI. This is a consequence of the high $P_{\text {on }}$ required to sustain auto-oscillation for M5 and ${ }_{245}$ MRI. An overview of operating conditions for $V_{L}=0 \mathrm{~mL}$ in the $\left(f_{0, d}, \bar{P}_{u}\right)$ pa- 
rameter space for deformable VF replicas is plotted in Fig. 7.

\subsection{Water spraying experiments}

During water spraying experiments, at first single phase airflow (dry condisurface tension,

$$
W e=\frac{\rho_{L} U_{L}^{2} D_{n}}{\sigma}
$$

for water droplets with diameter $D_{n}$ issued by the spray yields $W e \approx O\left(10^{2}\right)$ with water-air surface tension $\sigma=72 \mathrm{mN} / \mathrm{m}$. It follows that the injected liquid jet is expected to break up into much smaller droplets favoring initial mixing 270 itive $z$-direction in Fig. 6) so that a stable oscillatory pattern with oscillation frequency $f_{0, d}$ is established prior to water spraying using operating conditions detailed in Section 4.2.

Then, distilled water (motivated in Section 3) is injected manually at the downstream end of the channel, along the negative $z$-direction, by emptying a graduated (accuracy $0.5 \mathrm{~mL}$ ) syringe (diameter $14 \mathrm{~mm}$, maximum volume $11 \mathrm{~mL}$ ) equipped with a commercial round spray nozzle (diffusion angle $20^{\circ} \pm 1^{\circ}$, diameter $D_{n}=0.7 \pm 0.1 \mathrm{~mm}$, length $10 \mathrm{~mm}$ ) containing a known water volume $1 \leq V_{L} \leq 5 \mathrm{~mL}$. Water is nebulized homogeneously across the constricted area operating an electrical switch at the start and end of injection. The duration of water injection $\Delta t_{L}$ takes less than $3 \mathrm{~s}$ and water injection flow rate $\phi_{L}$ yields about $\phi_{L}=2 \mathrm{~mL} / \mathrm{s}$ [23]. It follows that liquid bulk velocity $U_{L}$ at the spray nozzle exit approximates $5.2 \mathrm{~m} / \mathrm{s}$ and the syringes' driving pressure in with the airflow. The droplet relaxation time

$$
t_{d}=\frac{\rho_{L} D_{n}^{2}}{18 \mu_{G}}
$$




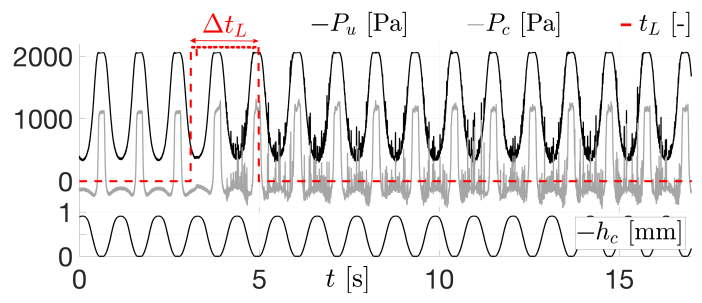

(a) MDR replica $V_{L}=4 \mathrm{~mL}, f_{c}=1 \mathrm{~Hz}$

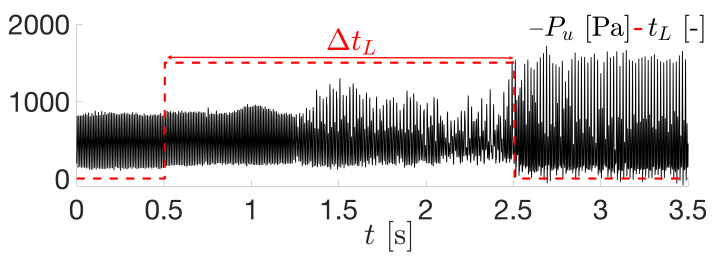

(b) PLT, $V_{L}=4 \mathrm{~mL}, f_{0, d} \approx 105 \mathrm{~Hz}$

Figure 8: Time traces of measured signals near water spraying with duration $\Delta t_{L}$ for: a) motion driven rigid MDR VF replica, b) deformable PLT VF replica for condition $(3300,0)$.

reduces from $t_{d}=1.5 \mathrm{~s}$ for $D_{n}=0.7 \mathrm{~mm}$ as droplets break up so that their diameter decreases. The Stokes number, characterising the behaviour of droplets suspended in the flow,

$$
S t k=\frac{t_{d} U_{G}}{L_{z}}
$$

varies with $U_{G}$ during a single oscillation cycle leading to mixing of the droplets with the flow in the case of low Stokes numbers (smaller than 1) or surface hydration due to droplet deposition along the upstream pipe (vocal tract) and vocal folds for larger Stokes numbers (larger than 1). It follows that the flow patterns vary during a single oscillation cycle. During full closure, water can accumulate on the surface of the vocal folds replicas along the downstream end resulting in a water film. Therefore, during the opening phase the flow might overcome surface tension at the air-water interface leading to different flow patterns during the closing and opening phase. The viscous contribution to the flow reduces as $A_{c}$ increases. 
driven rigid MDR VF replica $\left(P_{u}(t), P_{c}(t), h_{c}(t)\right.$ and $\left.t_{L}(t)\right)$ and deformable PLT VF replica $\left(P_{u}(t)\right.$ and $\left.t_{L}(t)\right)$ are illustrated in Fig. 8. Pressure signals gathered $5 \mathrm{~s}$ after water spraying is finished are quantitatively analysed in order to avoid the transitional flow associated with the injection. This way the impact of surface hydration due to an hydration spray usage is mimicked. It is verified that no water passes through the vocal folds during oscillation. Quantified pressure features are outlined in Section 5 .

\section{Pressure analysis}

\subsection{Oscillation features}

The influence of water spraying on the waveform shape is quantified by time tracking period $T$ and associated features within each pressure $P$. oscillation cycle as illustrated in Fig. 9 for upstream pressure $P .=P_{u}$ : maximum pressure $P_{\cdot, \text { max }}$, peak-to-peak pressure amplitude $\mathcal{A}$, closed portion $T^{t}$ which is composed of a closing $T^{c}$ and an opening $T^{o}$ component. Closed portion $T^{t}$ is defined as the duration of the period for which pressure $P$. exceeds a threshold value. The threshold value is set to $P_{u, \max }-0.95 \mathcal{A}$ for deformable VF replicas when upstream pressure $P_{u}$ is analysed $\left(P .=P_{u}\right)$ and to $P_{c, \max }-0.85 \mathcal{A}$ for motion driven MDR VF replica when constriction pressure $P_{c}$ is analysed $\left(P .=P_{c}\right)$. The part of $T^{t}$ during which $P .(t)$ increases corresponds to closing portion $T^{c}$ and the remaining decreasing part corresponds to opening portion $T^{o}$ so that $T^{t}=T^{c}+T^{o}$. Note that the ratio $T^{c} / T^{o}$ informs on closing-opening asymmetry within each cycle. Therefore $T^{c} / T^{o}$ allows to quantify the imprint of mean flow changes during these two phases, characterised by a changing closing-opening asymmetry. Such an asymmetry is likely as outlined in Section 4.1 and qualitatively described for the MDR repica in [23].

To identify properties for any of the aforementioned temporal feature vectors, for the sake of simplicity denoted $\mathcal{P}$ in general, its mean value $\overline{\mathcal{P}}=\frac{1}{N} \sum_{i=1}^{N} \mathcal{P}_{i}$ 


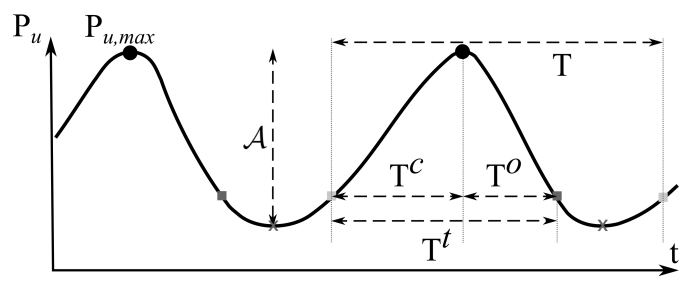

Figure 9: Illustration of waveform shape feature vectors for $f_{0}$ cycle-to-cycle analysis for pressure $P .=P_{u}$.

and its standard deviation

$$
\Delta \mathcal{P}=\sqrt{\frac{1}{N} \sum_{i=1}^{N}\left|\mathcal{P}_{i}-\overline{\mathcal{P}}\right|^{2}}
$$

are gathered where $N=50$ indicates the used number of periods. The perturbation intensity is then obtained as $\frac{\Delta \mathcal{P}}{\overline{\mathcal{P}}}$, the ratio between standard deviation $\Delta \mathcal{P}$ and mean value $\overline{\mathcal{P}}$. It is noted that when $\mathcal{P}$ represents a velocity time series this ratio yields the turbulence intensity. Rapid cycle-to-cycle perturbation $\zeta_{\mathcal{P}}$ is given as

$$
\zeta_{\mathcal{P}}=\frac{\frac{1}{N_{p}-1} \sum_{i=1}^{N_{p}-1}\left|\mathcal{P}_{i}-\mathcal{P}_{i+1}\right|}{\overline{\mathcal{P}}} .
$$

For peak-to-peak amplitude $\mathcal{A}$ (i.e. $\mathcal{P}=\mathcal{A}$ ) and period $T$ (i.e. $\mathcal{P}=T), \zeta_{\mathcal{A}}$ and $\zeta_{T}$ yield an estimation of common voice parameters shimmer and jitter, respectively $[2,13]$.

Other common voice parameters are derived from a harmonic decomposition of $P_{u}$. Signal-to-noise ratio SNR (in $\mathrm{dB}$ ) is computed as the ratio of the summed power of all signal harmonics $P_{\text {signal }}$ based on the first harmonic frequency $f_{N}$ to the summed power of the remaining noise $P_{\text {noise }}$ :

$$
S N R=10 \log _{10}\left(\frac{P_{\text {signal }}}{P_{\text {noise }}}\right) .
$$

Total harmonic distortion rate $T H D$ (in $\mathrm{dB}$ ) is computed from the ratio between the summed power of higher harmonics frequencies $P_{\text {harm }}$ and the power of the 


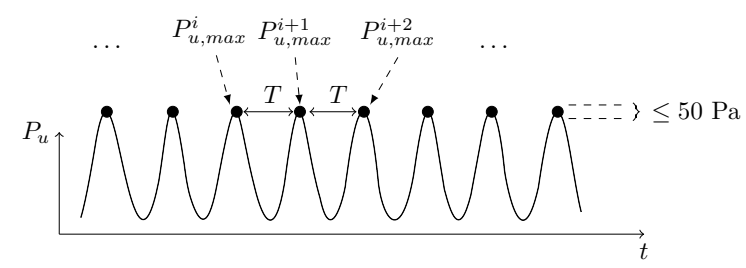

(a) Normal oscillation cycle regime, $f_{N}=f_{0}$

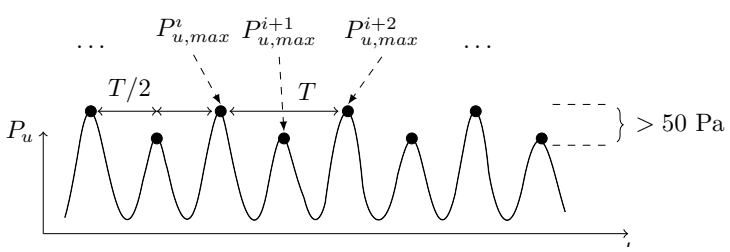

(b) Subharmonic oscillation cycle regime, $f_{N}=f_{0} / 2$

Figure 10: Illustration of $f_{N}$ cycle analysis and oscillation regimes: a) normal $f_{N}=f_{0}, \mathrm{~b}$ ) subharmonic $f_{N}=f_{0} / 2$.

first harmonic frequency $P_{f_{N}}$ :

$$
T H D=10 \log _{10}\left(\frac{P_{h a r m}}{P_{f_{N}}}\right) .
$$

Consequently, the signal quality is said to improve for increasing $S N R$ and decreasing $T H D$ since it indicates less noise and less harmonic distortion, respectively.

\subsection{Oscillation cycle-to-cycle analysis: $f_{0}$ and $f_{N}$}

Classically, first harmonic frequency $f_{N}$ corresponds to oscillation frequency $f_{0}$ of each normal oscillation cycle given as $1 / T$ where period $T$ corresponds to the time interval between successive waveform maxima: $P_{u, \max }(i)$ and $P_{u, \max }(i+$ 1) as shown in Fig. 10(a).

An alternative subharmonic oscillation cycle regime is observed when

$$
\left\{\begin{array}{l}
\left|P_{u, \max }^{i}-P_{u, \text { max }}^{i+1}\right|>50 \mathrm{~Pa} \\
\left|P_{u, \max }^{i}-P_{u, \max }^{i+2}\right| \leq 50 \mathrm{~Pa}
\end{array}\right.
$$


as depicted in Fig. 10(b). In this case, first harmonic frequency $f_{N}$ corresponds to subharmonic frequency $f_{0} / 2$ associated with period $T$ between maximum $P_{u, \max }^{i}$ and $P_{u, \max }^{i+2}$. The used threshold of $50 \mathrm{~Pa}$ corresponds to $10 \%$ up to $20 \%$ of $\bar{P}_{u}$ for the PLT VF replica and $<10 \%$ of $\bar{P}_{u}$ for silicone VF replicas (EPI, MRI and M5) (Table 3).

In summary, first harmonic frequency $f_{N}$ of each cycle yields:

$$
f_{N}= \begin{cases}f_{0} / 2, & \text { if }\left|P_{u, \max }^{i}-P_{u, \max }^{i+1}\right|>50 \mathrm{~Pa} \\ f_{0}, & \text { otherwise. }\end{cases}
$$

Consequently, besides a cycle-to-cycle analysis associated with $f_{0}$, a cycle-tocycle analysis associated with $f_{N}$ can be performed when a subharmonic oscillation cycle regime is detected.

\subsection{Period change under the added mass hypothesis}

The normal cycle period $T$, and hence oscillation frequency $f_{0}=1 / T$, characterising the auto-oscillation due to the FS interaction described by Eq. (1), is dependent on the mechanical resonance frequency, determined by its elasticity and its mass as $T \propto \sqrt{\frac{m}{k}}[20,22]$. It is assumed that water spraying does not affect the VF's elasticity so that $k$ remains constant. This is a reasonable working assumption since water does not penetrate the VF replicas. On the other hand, $m$ increases under the hypothesis that spraying water results in adding mass $m_{L}$ to the vibrating mass $m, e . g$. due to accumulation of water on the downstream end of the VF replica during the closed phase as argued in Section 4.1.

The added mass is less than the mass of the injected water so that $m_{L}=$ $\alpha_{L} m_{V_{L}}$ with $\alpha_{L} \leq 1$ holds and the same way vibrating mass $m$ is less than total vocal fold mass $m_{d}$ so that $m=\alpha_{m} m_{d}$ with $\alpha_{m}<1$ holds as well. Thus, when $m_{L}$ is added to vibrating mass $m$, normal oscillation period $T$ deflects from its value $T_{d}$ for $V_{L}=0 \mathrm{~mL}$ as:

$$
\frac{T}{T_{d}}=\sqrt{1+\frac{\alpha_{L}}{\alpha_{m}} \frac{m_{V_{L}}}{m_{d}}} \quad \text { so that } \quad T \geq T_{d}
$$


It follows from (15) that added mass results an increase of the ratio $\frac{T}{T_{d}}$ so that the period increases and hence the oscillation frequency decreases.

The magnitude of the increase is estimated setting $\alpha_{L}=1$ and $\alpha_{m}=1$. This way both $m_{L}$ and $m$ are overestimated so that their ratio provides a first estimation of the tendency expected under the added mass hypothesis. From Table 1 (for $V_{L}=0 \mathrm{~mL}$ ) it is seen that the mean mass of all deformable $\mathrm{VF}$ replicas yields $\bar{m}_{d}=2.02 \mathrm{~g}$ and its standard deviation $\Delta m_{d}=0.22 \mathrm{~g}(11 \%)$.

\section{Results for water spraying $V_{L}>0 \mathrm{~mL}$}

\subsection{Flow through the glottal constriction}

Measured upstream pressure $P_{u}(t)$, constriction pressure $P_{c}(t)$, water spraying time tag $t_{L}(t)$ and imposed minimum spacing $h_{c}(t)$ for $V_{L}=4 \mathrm{~mL}$ and forcing frequency $f_{c}=1 \mathrm{~Hz}$ near the instant of water spraying are plotted in Fig. 8(a). In [23] it was qualitatively shown that when water was sprayed $\left(V_{L}>0 \mathrm{~mL}\right)$ viscous two-phase mixing affects $P_{c}(t)$ near closure, i.e. during both the opening and closing phase of the oscillation, which results in increased amplitude $\mathcal{A}$, extended duration of pressure increase near closure $T^{t}$ and different flow regimes in the opening and closing phase affecting closing-opening asymmetry $T^{c} / T^{o}$. Quantitative evidence of the effect of $V_{L}$ on $\mathcal{A}, T^{t}$ and $T^{c} / T^{o}$ with respect to their values for single-phase airflow $\mathcal{A}_{d}, T_{d}^{t}$ and $\left(T^{c} / T^{o}\right)_{d}$ (subscript $d, V_{L}=0 \mathrm{~mL}$ ) is provided in Fig. 11(a), Fig. 11(b) and Fig. 11(c).

Both mean (symbols) and standard deviation (vertical error bars) of $\mathcal{A}$ and $T^{t}$ augments with $V_{L}$ up to at least $10 \%$. The standard deviation of $T^{c} / T^{o}$ increases with $V_{L}$ as well whereas mean $T^{c} / T^{o}$ first increases $V_{L} \in\{1,2\} \mathrm{mL}$ and then decreases for $V_{L}>2 \mathrm{~mL}$ up to $>15 \%$ so that opening-closing asymmetry of the glottal flow qualitatively described and modelled in [23] is quantitatively confirmed. The increased perturbation points to an increased complexity and fluctuation of $P_{c}$ whose origin needs yet to be fully determined, although droplet 


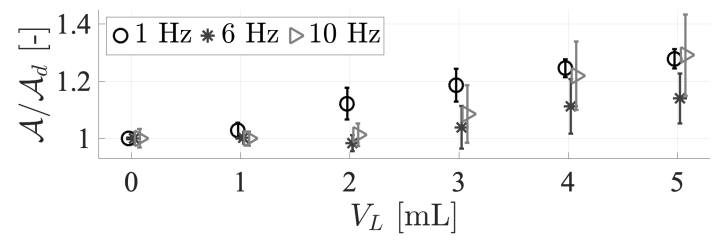

(a) $\mathcal{A} / \mathcal{A}_{d}$

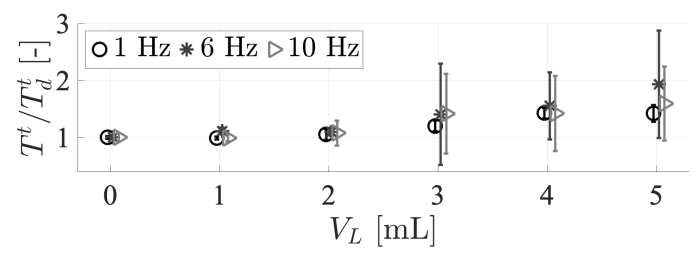

(b) $T^{t} / T_{d}^{t}$

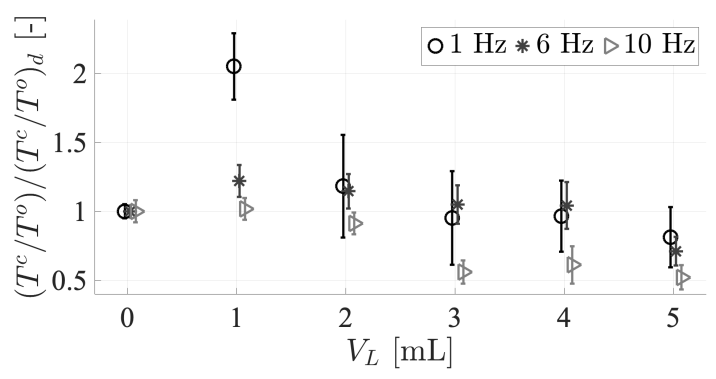

(c) $\left(T^{c} / T^{o}\right) /\left(T^{c} / T^{o}\right)_{d}$

Figure 11: Motion driven rigid MDR VF replica: a) $\mathcal{A}\left(V_{L}\right) / \mathcal{A}_{d}$ from $P_{c}(t)$, b) $T^{t}\left(V_{L}\right) / T_{d}^{t}$ from $P_{c}(t)$ and c) $\left(T^{c} / T^{o}\right) /\left(T^{c} / T^{o}\right)_{d}$ from $P_{c}(t)$. Subscript $d$ indicates $V_{L}=0 \mathrm{~mL}$. Mean (symbols) and standard deviation (vertical bars) are plotted in a), b) and c). For clarity data for $\{1,10\} \mathrm{Hz}$ are shifted around $V_{L}$. 


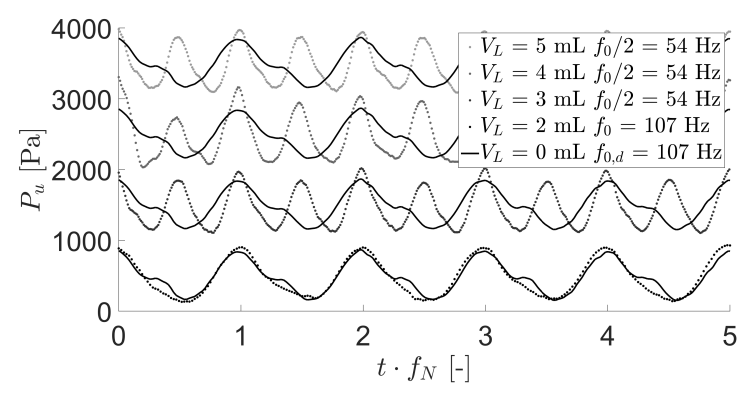

(a) PLT, $P_{u}\left(t \cdot f_{N}\right)$ for $V_{L} \leq 5 \mathrm{~mL}$

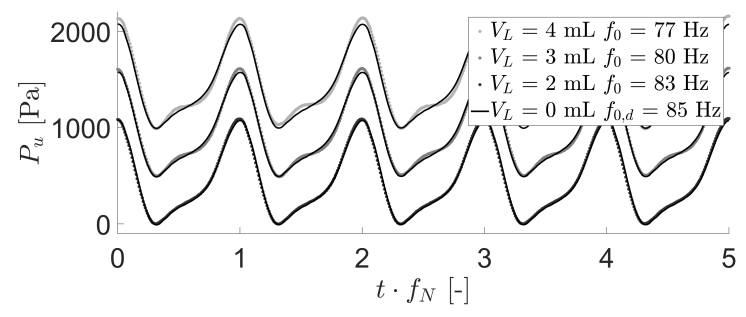

(b) EPI, $P_{u}\left(t \cdot f_{N}\right)$ for $V_{L} \leq 5 \mathrm{~mL}$

Figure 12: a) $P_{u}\left(t \cdot f_{N}\right)$ for PLT condition $(3300,0)$ and $\left.V_{L} \leq 5, \mathrm{~b}\right) P_{u}\left(t \cdot f_{N}\right)$ for EPI and $V_{L} \leq 5$. Time is normalised with $f_{N}$ yielding $f_{0(, d)}$ or $f_{0} / 2$. Curves for $V_{L} \geq 3 \mathrm{~mL}$ are upshifted, $V_{L} \geq 2 \mathrm{~mL}$ (dotted lines) and $V_{L}=0 \mathrm{~mL}$ (full line).

375 induced turbulence is suggested in [23].

\subsection{FSI during auto-oscillation}

\subsubsection{Measured upstream pressure}

A typical measurement of upstream pressure $P_{u}(t)$ and water spraying tag $t_{L}(t)$ near the instant of water spraying for a deformable VF replica (PLT) is plotted in Fig. 8(b). The influence of water spraying on time traces of upstream pressure signal $P_{u}$ taken $5 \mathrm{~s}$ after water spraying is shown in more detail in Fig. 12(a) (pressurized tube VF replica PLT for $(3300,0): P_{P L T}=3300 \mathrm{~Pa}$, screw opening $0 \mathrm{~mm}$ ) and in Fig. 12(b) (silicone VF replica: EPI) where $P_{u}$ before $\left(V_{L}=0 \mathrm{~mL}\right.$, full line) and after $\left(V_{L}>0 \mathrm{~mL}\right.$, dotted line) water spraying is plotted. Note that for clarity pressure signals $P_{u}$ for $V_{L} \geq 3 \mathrm{~mL}$ are upshifted. 
The first harmonic frequency $f_{N}$ (Section 5.2) is used for time normalization and varies with $V_{L}$ as indicated in the legends. Note that depening on $V_{L}$ a subharmonic oscillation cycle regime becomes apparent for the PLT replica. The amplitude of $P_{u}$ is affected although the influence is small for EPI (Fig. 12(b)) and more prominent for PLT (Fig. 12(a)).

\subsubsection{Cycle-to-cycle analysis associated with $f_{0}$}

The normal oscillation cycle regime, determined by oscillation frequency $f_{0}$,

395 as a function of $V_{L}$ in order to assess the impact of water spraying on cycle-tocycle characteristics associated with $f_{0, d}$, i.e. for $V_{L}=0 \mathrm{~mL}$. For clarity, features for different deformable replicas are plotted in an interval centered around each $V_{L}$ value.

400

\section{Period T:}

- Mean and standard deviation of period $T$ associated with oscillation frequency $f_{0}$ are presented in Fig. 13 as a function of water volume $V_{L}$. Plotted values are normalised with mean period $T_{d}$ for $V_{L}=0 \mathrm{~mL}$.

405

Mean values (Fig. 13(a)) for EPI and MRI show a slight (less than 10\%) increase with $V_{L}$. For M5 and PLT $\left(P_{P L T}, o_{m m}\right)$-conditions mean $T / T_{d} \approx 1$ so that oscillation frequency $f_{0}$ is mostly unaffected except for condition $(2800,2)$, with the largest assessed initial opening $o_{m m}=2 \mathrm{~mm}$, for which $T$ increases with $\approx 35 \%$ when $V_{L} \geq 2 \mathrm{~mL}$. Observed $T / T_{d}$ do not agree with the gradual and significant (up to 100\%) increase predicted using Eq. (15) with $\alpha_{m}=1$ and $\alpha_{L}=1$. It follows that the added mass hypothesis (Section 5.3) is rejected as it can not explain $T / T_{d}$ so that observed changes to the oscillation period (and hence frequency) are not attributed to changes of the vibrating mass $m$.

The standard deviation of $T$ (error bars in Fig. 13(a)) and cycle-to-cycle ${ }_{415}$ perturbation $\zeta_{T}$ (Fig. 13(b)) increase with $V_{L}$. The increase is limited $(<10 \%)$ 


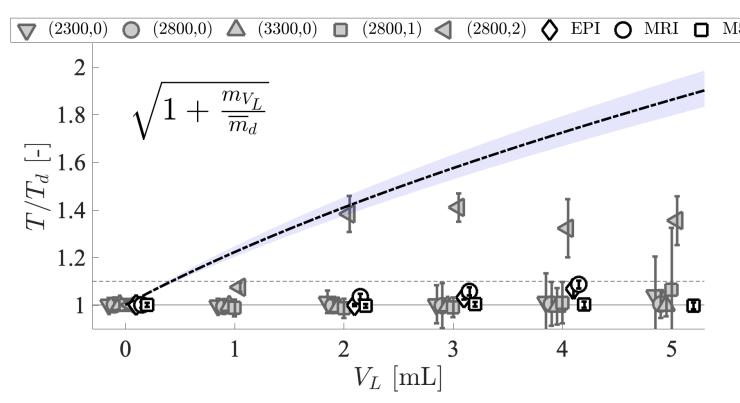

(a) $T / T_{d}: 1$ (full line), 1.1 (dashed line)

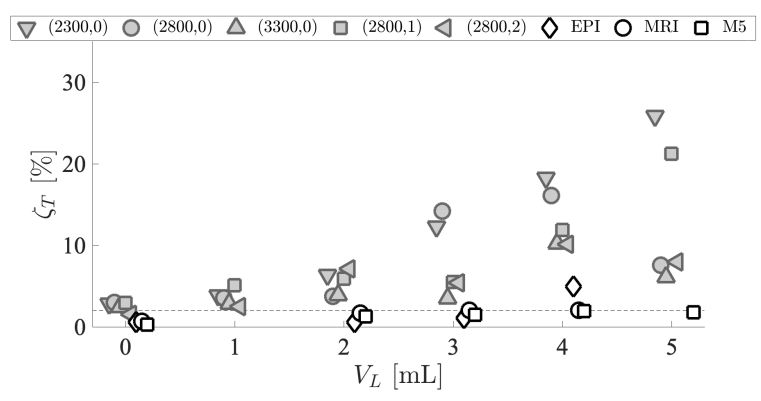

(b) $\zeta_{T}: 2 \%$ (dashed line)

Figure 13: Mean (symbols) and standard deviation (vertical bars) as a function of sprayed water volume $V_{L}$ : a) normalized period $T / T_{d}$ and tendency resulting from added mass hypothesis (dash-dotted line) with $m_{d}=\bar{m}_{d}$ (Eq. (15) for $\alpha_{L}=1$ and $\alpha_{m}=1$ ) and $m_{d}=\bar{m}_{d} \pm \Delta m_{d}$ (shaded region), b) period perturbation $\zeta_{T}$. Values from $f_{0}$ cycle-to-cycle analysis. 
for silicone VF replicas EPI, MRI and M5 regardless of $V_{L}$ and is more pronounced (between $10 \%$ and $30 \%$ ) for $V_{L} \geq 2 \mathrm{~mL}$ in the case of PLT VF replicas. Overall, $\zeta_{T}$ increases as the PLT VF replica condition $\left(P_{P L T, o_{m m}}\right)$ becomes more elastic, i.e. decreasing $P_{P L T} \in\{2300,2800,3300\} \mathrm{Pa}$ and $o_{m m}=0 \mathrm{~mm}$. 420 No clear tendency is observed with respect to the initial aperture condition $o_{m m}$.

\section{Amplitude $\mathcal{A}$ and turbulence intensity:}

- Mean and standard deviation of auto-oscillation amplitude $\mathcal{A}$ as a function of water volume $V_{L}$ are shown in Fig. 14(a). Amplitude values are normalised with respect to $\mathcal{A}_{d}$, i.e. mean amplitude for $V_{L}=0 \mathrm{~mL}$. For the PLT replica conditions with limited period increase $\left(T / T_{d}\right.$ increase $\left.<10 \%\right)$, a large amplitude increase (between 10\% and 120\%) and large standard deviations (up to 40\%) are observed whereas they are less than $15 \%$ for the remaining PLT condition $(2800,2)$. For all silicone VF replicas standard deviations of $\mathcal{A}$ are negligible and the mean value increase is limited to $<10 \%$ for MRI and M5 and more pronounced up to $35 \%$ for EPI.

Cycle-to-cycle perturbation $\zeta_{A}$ (Fig. 14(b)) remains less than $2 \%$ for all silicone VF replicas and increases up to $60 \%$ for PLT replica conditions. The same tendency is observed when considering turbulence intensity $\Delta U_{P_{u, \max }} / \bar{U}_{P_{u, \max }}$ where $U_{P_{u, \max }}$ is estimated for each cycle using Eq. (5). The turbulence intensity yields less than $1 \%$ for all silicone VF replicas and increases with $V_{L}$ up to $15 \%$ for PLT replica conditions. Overall perturbation measures $\zeta_{A}$ and $\Delta U_{P_{u, \text { max }}} / \bar{U}_{P_{u, \text { max }}}$ increases as the PLT VF replica condition $\left(P_{P L T, o_{m m}}\right)$ becomes more elastic (decreasing $P_{P L T} \in\{3300,2800,2300\} \mathrm{Pa}$ and $o_{m m}=$

${ }_{440} 0 \mathrm{~mm}$ ) and as the initial aperture decreases (decreasing $o_{m m} \in\{2,1,0\} \mathrm{mm}$ and $\left.P_{P L T}=2800 \mathrm{~Pa}\right)$.

\section{Closing and opening $T^{t}=T^{c}+T^{o}$ :}

. Mean and standard deviation of $T^{t} / T$, expressing the ratio between the dura${ }_{445}$ tion of the pressure peak associated with closing and opening and period $T$, is 


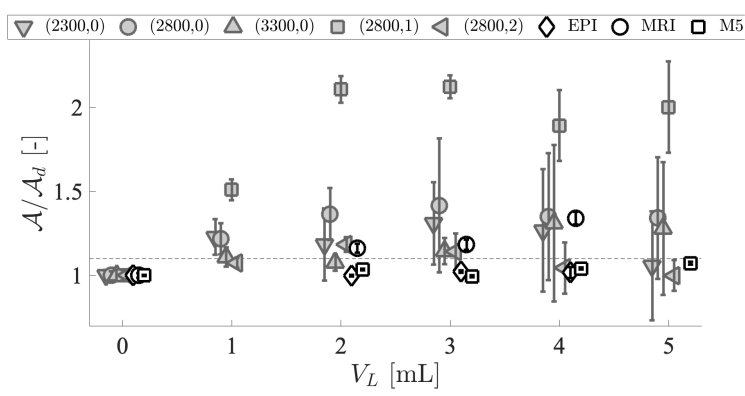

(a) $\mathcal{A} / \mathcal{A}_{d}: 1.1$ (dashed line)

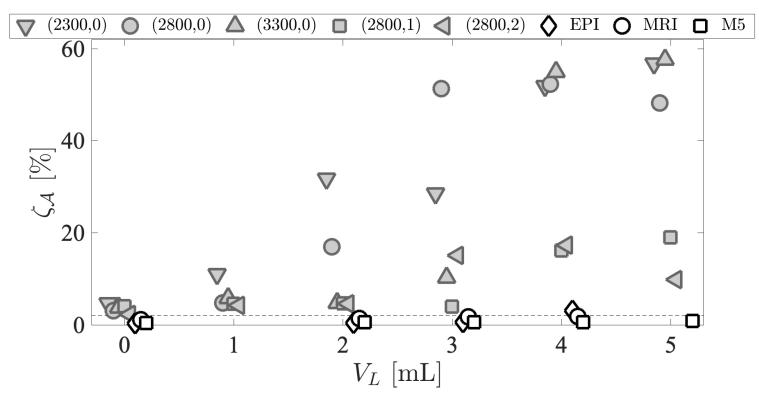

(b) $\zeta_{\mathcal{A}}: 2 \%$ (dashed line)

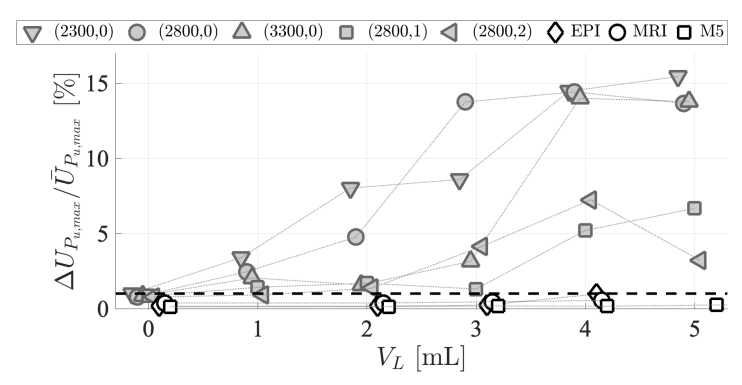

(c) $\Delta U_{P_{u, \max }} / \bar{U}_{P_{u, \max }}: 1 \%$ (dashed line)

Figure 14: Mean (symbols) and standard deviation (vertical bars) as a function of sprayed water volume $V_{L}$ : a) normalized amplitude $\mathcal{A} / \mathcal{A}_{d}$, b) amplitude perturbation $\zeta_{\mathcal{A}}$, c) turbulence intensity $\Delta U_{P_{u, \max }} / \bar{U}_{P_{u, \max }}$. 


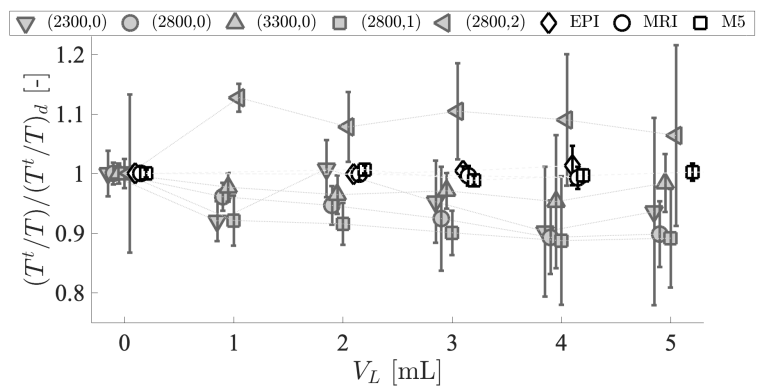

(a) $\left(T^{t} / T\right) /\left(T^{t} / T\right)_{d}: 1.0$ (dashed line)

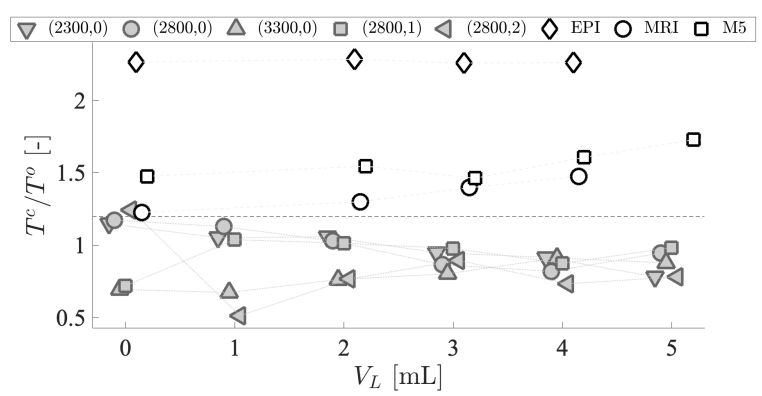

(b) $T^{c} / T^{o}: 1.2$ (dashed line)

Figure 15: Mean (symbols) and standard deviation (vertical bars) as a function of sprayed water volume $V_{L}$ : a) normalised closed portion $\left(T^{t} / T\right) /\left(T^{t} / T\right)_{d}$, b) closing-opening asymmetry $\left(T^{c} / T^{o}\right) /\left(T^{c} / T^{o}\right)_{d}$. 
plotted in Fig. 15. Shown values are normalised by $\left(T^{t} / T\right)_{d}$ for $V_{L}=0 \mathrm{~mL}$. For silicone replicas $T^{t} / T$ is unaffected by $V_{L}$ since $T^{t} / T \approx 1$ and standard deviations are less than $3 \%$. For PLT conditions it is seen that ratio $T^{t} / T$ decreases compared to its value for $V_{L}=0 \mathrm{~mL}$ while the standard deviation increases to $20 \%$. A different tendency is again observed for PLT condition $(2800,2)$ for which the ratio yields $\approx 10 \%$ for $V_{L}>0 \mathrm{~mL}$.

Closing-opening asymmetry $T^{c} / T^{o}$ associated with $T^{t}$ is plotted in Fig. 6.2.2. Different tendencies are observed for the silicone VF replicas and PLT replica conditions. For silicone VF replicas $T^{c} \geq 1.2 T^{o}$ and its value either remains (EPI for which $T^{c} / T^{o} \approx 2.3$ ) or increases with $V_{L}$ (MRI and M5) so that the closing phase dominates the opening phase regardless of $V_{L}$. The opposite tendency is found for the PLT VF replica conditions since the range for $V_{L}=0 \mathrm{~mL}$ $\left(T^{c} / T^{o} \in\left[\begin{array}{ll}0.7 & 1.2\end{array}\right]\right.$ reduces with $V_{L}$ to $T^{c} / T^{o} \approx 0.8 \pm 0.1$. Consequently, for PLT replica conditions the opening phase is more prominent than the closing phase. Note that these tendencies can be qualitatively observed from upstream pressure time signals plotted in Fig. 12(a) for PLT condition $(3300,0)$ and in Fig. 12(b) for the silicone EPI VF replica.

\subsubsection{Cycle-to-cycle analysis associated with $f_{N}$}

Evidence of subharmonic oscillation regime:

- The first harmonic frequency $f_{N}$ is determined as outlined in Section 5.2. For silicone vocal folds replicas (EPI, MRI and M5) the first harmonic frequency (Eq. (14)) equals the harmonic frequency $\left(f_{N}=f_{0}\right)$ for all $V_{L}$ (see e.g. Fig. 12(b) for the EPI VF replica), so that the power ratio between subharmonic frequency $f_{0} / 2$ and harmonic frequency $f_{0}$ yields zero, i.e. $P_{f_{0} / 2} / P_{f_{0}}=0$, and hence $1-P_{f_{0} / 2} / P_{f_{0}}=1$ (or $100 \%$ ). For PLT VF conditions on the other hand, first harmonic frequency $f_{N}$ shifts from harmonic frequency $f_{0}$ to subharmonic frequency $f_{0} / 2$ as $V_{L}$ increases, which is illustrated for $(3300,0)$ in Fig. 12(a). ${ }_{475}$ Values of $1-P_{f_{0} / 2} / P_{f_{0}}$ for all PLT replica conditions as a function of $V_{L}$ are 


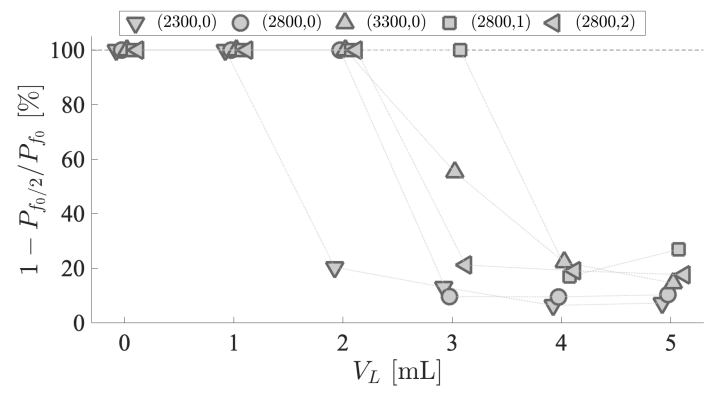

(a) $1-P_{f_{0} / 2} / P_{f_{0}}: 100 \%$ (dashed line)

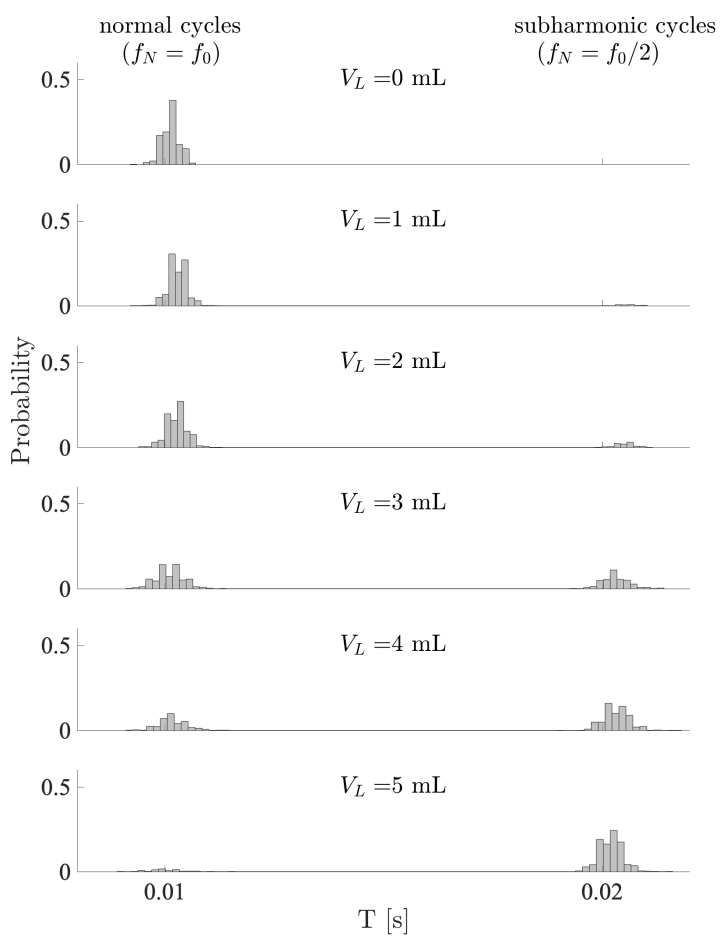

(b) $T$ probability for $(2800,0)$

Figure 16: Evidence for subharmonic frequency $f_{0} / 2$ as a function of $V_{L}$ : a) subharmonic-toharmonic power ratio $1-P_{f_{0} / 2} / P_{f_{0}}$, b) probability of $T$ from $f_{N}$ cycle-to-cycle analysis for PLT condition $(2800,0)$. 
plotted in Fig. 16(a). The ratio decreases from $100 \%$ for $V_{L}=0 \mathrm{~mL}$, when no subharmonic is present and $f_{N}=f_{0}$ holds, to less than $30 \%$ for $V_{L} \geq 4 \mathrm{~mL}$. The rate at which first harmonic $f_{N}$ shifts from harmonic frequency $f_{0}$ to subharmonic $f_{0} / 2$ as $V_{L}$ increases depends on the assessed PLT replica condition

${ }_{480}\left(P_{P L T}, o_{m m}\right)$. Note that since $1-P_{f_{0} / 2} / P_{f_{0}}>0$, it implies that in all cases harmonic frequency $f_{0}$ has more energy than subharmonic frequency $f_{0} / 2$.

\section{Period T:}

. Fig. 16(b) shows the probability distribution of $T$ as a function of $V_{L}$ for PLT condition $(2800,0)$. Period $T$ is detected as outlined in Section 5.2. Two distribution peaks are observed. The first peak associated with $f_{N}=f_{0}$ for $T \approx 0.01 \mathrm{~s}$ is dominant for $V_{L}<3 \mathrm{~mL}$ and the second peak associated with $f_{N}=f_{0} / 2$ for $T \approx 0.02 \mathrm{~s}$ is dominant for $V_{L}>3 \mathrm{~mL}$. For $V_{L}=3 \mathrm{~mL}$ the maximum probability of both peaks is similar. Furthermore, it is noted that both peaks have similar width indicating that the variation around the mean peak value is similar as well.

A cycle-to-cycle analysis for $T / T_{d}$ associated with first harmonic frequency $f_{N}$ is plotted in Fig. 17(a) and associated cycle-to-cycle perturbation $\zeta_{T}$ is shown in Fig. 17(b). For silicone VF replicas (M5, MRI, EPI) $f_{N}=f_{0}$ so that plotted values are similar to those in Fig. 13(a). For PLT replica conditions first harmonic frequency $f_{N}$ equals either harmonic frequency $f_{0}$ or subharmonic frequency $f_{0} / 2$ as illustrated in Fig. 16(b) for PLT condition $(2800,0)$. Consequently, mean $T / T_{d}$-values gradually increase with $V_{L}$ towards $T / T_{d} \approx 2$ expressing the growing weight of subharmonic frequency $f_{0} / 2$ on mean values of $T / T_{d}$ as is illustrated in Fig. 16.

Overall, for PLT replica conditions standard deviations of $T / T_{d}$ (Fig. 17(a)) and cycle-to-cycle perturbations $\zeta_{T}$ (Fig. 17 (b)) for $V_{L} \geq 1 \mathrm{~mL}$ associated with a $f_{N}$ cycle-to-cycle analysis are increased compared to those obtained from a $f_{0}$ cycle-to-cycle analysis (Fig. 13). This increase is among others due to the first 


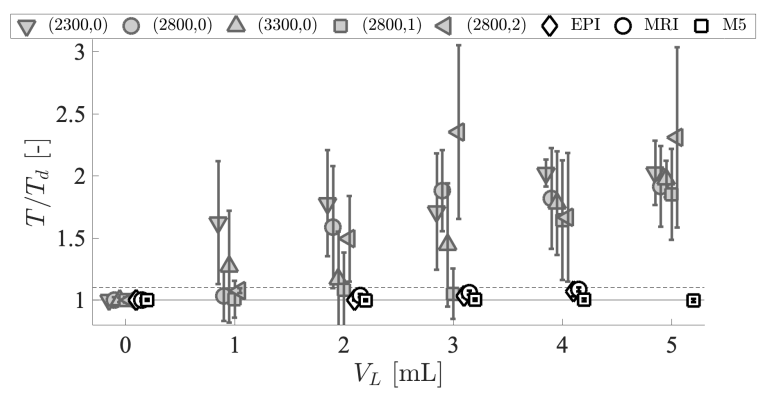

(a) $T / T_{d}: 1$ (full line), 1.1 (dashed line)

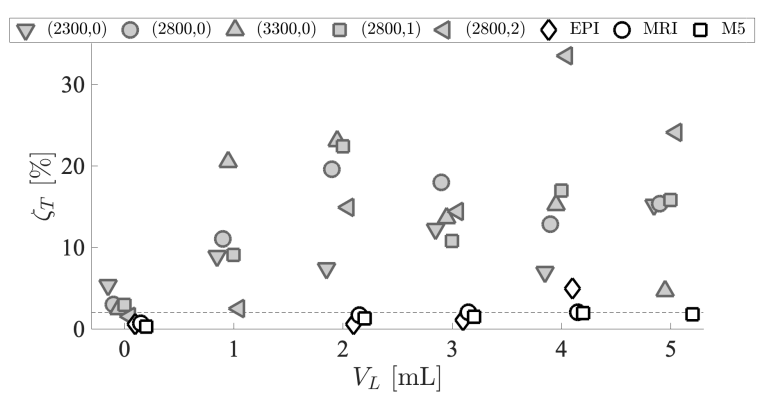

(b) $\zeta_{T}: 2 \%$ (dashed line)

Figure 17: Mean (symbols) and standard deviation (vertical bars) as a function of sprayed water volume $V_{L}$ : a) normalized period $T / T_{d}$, b) period perturbation $\zeta_{T}$. Values from $f_{N}$ cycle-to-cycle analysis. 


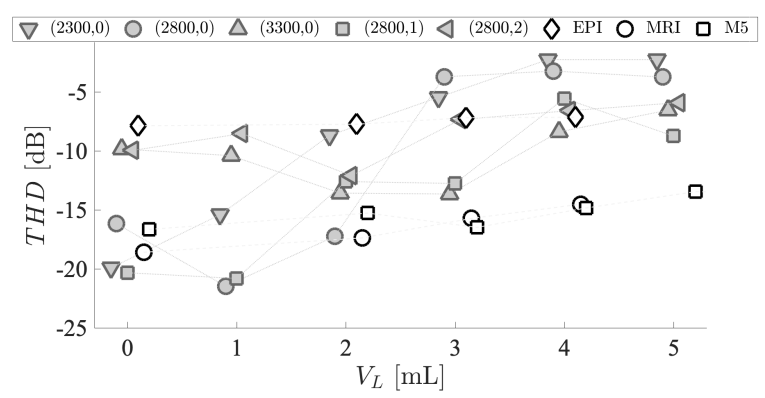

(a) $T H D$

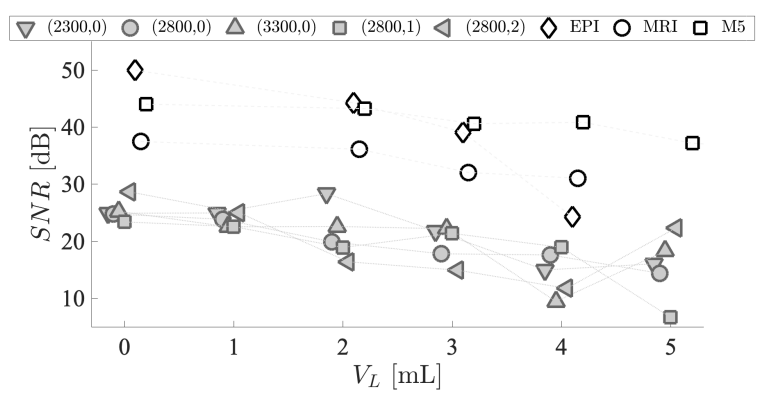

(b) $S N R$

Figure 18: Overall spectral features: a) total harmonic distortion rate $T H D$, b) signal-to-noise ratio $S N R$.

harmonic frequency $f_{N}$ shifting between $f_{0}$ and $f_{0} / 2$ (see Fig. 16(b)).

Overall spectral features THD and $S N R$ :

- Changes to the harmonic contents with respect to the lowest first harmonic frequency $f_{N}$ of upstream pressure $P_{u}$ with $V_{L}$ are further quantified by the total harmonic distortion rate THD (Eq. (12)) depicted in Fig. 18(a). For silicone replicas (EPI, MRI and M5) the increase is limited since it is most notable for MRI ( $\approx 4 \mathrm{~dB}$ increase). For PLT replica conditions an overall increase $(>4 \mathrm{~dB})$ with $V_{L}$ is observed. Nevertheless, the increase is not monotonous since for $0<V_{L} \leq 3 \mathrm{~mL}$ the subharmonic frequency $f_{0} / 2$ gradually imprints on the harmonic composition with a rate depending on initial conditions $\left(P_{P L T}, o_{m m}\right)$. Overall, it is seen that $T H D$ increases with $V_{L}$ for all VF replicas so that for 
all VF replicas harmonics are generated with $V_{L}$.

From Fig. 18(b) is seen that the signal-to-noise ratio $S N R$ (Eq. (11)) decreases as $V_{L}$ increases for all replicas. The overall decrease yields $\geq 10 \mathrm{~dB}$ except for MRI and M5 for which the decrease is limited to $\leq 5 \mathrm{~dB}$. It is noted that for all $V_{L}, S N R$-values for silicone VF replicas are greater than the ones for PLT VF replica conditions.

\section{Discussion}

For all deformable VF replicas, increasing $V_{L}$ in the range $V_{L} \leq 5 \mathrm{~mL}$ affects the mean waveform shape of the upstream pressure during normal oscillation regime: increased oscillation cycle period $T$, increased amplitude $\mathcal{A}$ and altered closing and opening portions $T^{c, o, t}$ so that closing-opening asymmetry is affected. The perturbation of this mean waveform shape increases with $V_{L}$ as well: increased total harmonic distortion rate $T H D$, decreased signal-to-noise ratio $S N R$ and increase of both fast $\left(\zeta_{T, \mathcal{A}}\right)$ and overall (standard deviations) fluctuations of waveform shape features. Therefore, water spraying affects the normal auto-oscillation regime resulting in changes to the mean waveform shape - increased oscillation period and amplitude, changes to opening and closing portions - and increased perturbation of this mean waveform.

A preliminary qualitative description of the effect of water spraying on the glottal flow through the motion driven MDR replica [23] was confirmed quantitatively in this work for waveform amplitude $\mathcal{A}$ and closing and opening portions $T^{t, c, o}$. Found tendencies on the motion driven VF replica compare well with general normal oscillation cycle tendencies described for deformable VF replicas: changes to mean values (amplitude increase and altered opening and closing portions $T^{t, c, o}$ ) and increased perturbation. Therefore, current findings support that flow changes following two-phase water-air mixing observed for the motion 
driven MDR replica do also occur in the case of the deformable VF replicas. Flow changes following two-phase water-air mixing will alter pressure forces $\left(F_{A}\right.$ right-hand side of Eq. 1) on the enveloping VF structure and hence contribute to observed waveform shape changes. Current data disprove that the vibrating mass increases with $V_{L}$. Therefore, $m$ in the left-hand side of Eq. (1) can be assumed independent of $V_{L}$ and mass changes can not explain observed changes to normal oscillation period $T$ or frequency $f_{0}$. Hence, it is of interest for further research to account for flow mixing when aiming to model the FS interaction after surface hydration with a spray and to assess how far such a flow model can explain observed general tendencies of the impact of $V_{L}$ on the waveform shape associated with $V_{L}=0 \mathrm{~mL}$.

A part from mass $m$, initial conditions related to the elasticity and initial glottal aperture $\left(r, k\right.$, and $A_{0}$ left-hand side of Eq. (1)) as well as imposed mean upstream air pressure $\bar{P}_{u}\left(F_{A}\right.$ right-hand side of Eq. (1)) determine the FS interaction. Different deformable VF replicas were used resulting in a considerable variation of these initial conditions. Quantified waveform shape features showed the strong impact of initial conditions on the degree to which water spraying affects the mean waveform shape and its perturbation. In general, mean and even more so perturbation features are less affected by water spraying for silicone VF replicas (EPI, MRI and M5) than for PLT VF replica conditions. Large differences are observed between different PLT conditions as well. It is noted that the silicone M5 VF replica is only marginally affected by $V_{L}$. In future, a more systematic study is needed to quantify the effect of water spraying while varying initial conditions.

A subharmonic oscillation regime is generated by water spraying for $V_{L} \geq$ $2 \mathrm{~mL}$ in the case of PLT VF replica conditions and no subharmonic oscillation 575 regime is observed for silicone VF replicas. The generation of a sub-harmonic frequency point to non-linearities introduced following water spraying for the PLT VF replica. These non-linearities are potentially due to perturbations in- 
troduced by turbulence, estimated to increases with $V_{L}$ between $1 \%$ and $15 \%$ for the PLT VF replica whereas it remains less than $1 \%$ for silicone VF replicas. Further evidence is needed to sustain this statement.

General tendencies observed for the normal oscillation regime agree with findings reported in literature concerning the effect of surface hydration on human voice features $[12,17,2]$ and on excised larynges [13]: decrease of oscillation frequency $f_{0}$, changes to closing-opening asymmetry and to perturbation measures $\zeta_{A, T}$ as well as signal-to-noise ratio $S N R$ and total harmonic distortion rate $T H D$. Note that the shown influence of initial conditions observed for deformable VF replicas might partly explain the variety of tendencies observed on human speakers.

\section{Conclusion}

The effect of water spraying on a stable oscillation is quantified for increasing water volume. For deformable replicas, the mean upstream pressure waveform shape exhibits decreased oscillation frequency, increased amplitude, changing closing-opening asymmetry and increased perturbation. The degree to which these changes occur depends on initial conditions. These tendencies are consistent with quantified glottal pressure waveform shape changes observed for a motion driven rigid VF replica subjected to water spraying. This suggests that, as observed for the motion driven rigid replica, changing glottal flow behaviour due to air-water mixing underlies observed tendencies for the deformable VF replicas. Modeling studies are needed in order to assess to which extent mixing flow can explain current findings. In addition, further experimental research is needed in order to systematically consider the effect of initial replica conditions and their surface wettability, the influence of the used nozzle, quantify the droplet distribution during oscillation and further investigate physical mechanisms underlying the observed effects of water spraying on oscillation features. 


\section{Acknowledgements}

This work was partly supported by ArtSpeech project (ANR-15-CE23-0024).

Authors are grateful to Dr. Isao T. Tokuda (Ritsumeikan Univ., Japan) for helping us building silicone VF replicas.

\section{References}

[1] J. Gartner-Schmidt, Voice Therapy for the treatment of voice disorders, 4th Edition, Vol. 1 of Head \& Neck Surgery - Otolaryngology, Lippincott Williams \& Wilkins, Philadelphia, USA, 2006.

[2] C. Leydon, M. Sivasankar, D. Falciglia, C. Atkins, K. Fisher, Vocal fold surface hydration: a review, J Voice 23 (6) (2009) 658-665.

[3] K. Verdolini, I. Titze, A. Fernell, Dependence of phonatory effort on hydration level, J. Speech Hear Res 37 (1994) 1001-1007.

${ }_{620}$ [4] M. Sivasankar, T. Carroll, A. Kosinski, C. Rosen, Quantifying the effects of altering ambient humidity on ionic composition of vocal fold surface fluid, Laryngoscope 123 (2013) 1275-1278.

[5] N. Solomon, M. DiMatta, Effects of a vocally fatigue task and systemic hydration on phonation pressure threshold, J Voice 14 (2000) 341-362.

${ }_{625}$ [6] E. Santana, M. Masson, T. Araujo, The effect of surface hydration on teacher's voice quality: an intervention study, J Voice 31 (2017) 5-11.

[7] E. Erickson-Levendoski, M. Sivasankar, Investigating the effects of caffeine on phonation, J Voice 25 (2011) 215-219.

[8] M. Sivasankar, E. Erickson-Levendoski, Influence of obligatory mouth 630 breathing during realistic activities, on voice measures, J Voice 26 (2012) $9-13$. 
[9] K. Fisher, J. Ligon, J. Sobecks, D. Roxe, Phonatory effects of body fluid removal, J Speech Lang Hear Res 44 (2001) 354-367.

[10] A. Preetha, R. Banerjee, Comparison of artificial saliva substitutes, Trends Biomater. Artif. Organs 18 (2005) 178-186.

[11] K. Tanner, N. Roy, R. Merrill, K. Kendall, K. Miller, D. Clegg, A. Heller, D. Houtz, M. Elstad, Comparing nebulized water versus saline after laryngeal desiccation challenge in Sjögren's Syndrome, Laryngoscope 123 (2013) 2787-2792.

[12] M. Ayache, S. Ouaknine, P. Dejonkere, P. Prindere, A. Giovanni, Experimental study of the effects of surface mucus viscosity on the glottic cycle, J Voice 18 (2004) 107-115.

[13] M. Dollinger, F. Grohn, D. Berry, U. Eysholdt, G. Luegmaira, Preliminary results on the influence of engineered artificial mucus layer on phonation, J. Speech, Language, Hearing Res. 57 (2014) 637-647.

[14] J. Jiang, J. Ng, D. Hanson, The effects fo rehydration on phonation in excised canine larynges, J Voice 83 (1999) 1536-1552.

[15] N. Roy, K. Tanner, S. Gray, M. Blomgren, K. Fisher, An evaluation of the effects of three laryngeal lubricants on phonation threshold pressure (PTP), J Voice 17 (2003) 331-342.

[16] C. Leydon, M. Wroblewski, N. Eichorn, M. Sivasankar, A meta-analysis of outcomes of hydration intervention on phonation threshold pressure, J Voice 24 (2010) 637-643.

[17] M. Alves, E. Kruger, B. Pillay, K. van Lierde, J. van der Linde, The effect of hydration on voice quality in adults: a systematic review, J Voice 33 (2019) 13-28.

[18] C. Rosen, C. Simpson, Operative techniques in laryngology, SpringerVerlag, 2008. 
[19] I. Titze, The physics of small-amplitude oscillation of the vocal folds, J Acous Soc Am 83 (1988) 1536-1552.

[20] N. Ruty, X. Pelorson, A. Van Hirtum, I. Lopez, A. Hirschberg, An in-vitro setup to test the relevance and the accuracy of low-order models of the vocal folds, J Acous Soc Am 121 (2007) 479-490.

[21] F. Avanzini, Simulation of vocal fold oscillation with a pseudo-one-mass physical model, Speech Comm. 50 (2008) 95-108.

[22] P. Luizard, X. Pelorson, Threshold of oscillation of a vocal folds replica with unilateral surface growths, J Acous Soc Am 141 (2017) 3050-3058.

[23] A. Van Hirtum, A. Bouvet, X. Pelorson, Pressure drop for adiabatic airwater flow through a time-varying constriction, Phys Fluids 30 (2018) 101901.

[24] J. Cisonni, A. Van Hirtum, X. Pelorson, J. Willems, Theoretical simulation and experimental validation of inverse quasi one-dimensional steady and unsteady glottal flow models, J Acous Soc Am 124 (2008) 535-545.

[25] B. Pickup, S. Thomson, Flow-induced vibratory response of idealized versus magnetic resonance imaging-based synthetic vocal fold models, J Acous Soc Am 128 (2010) 124-129.

[26] P. Murray, S. Thomson, Vibratory responses of synthetic, self-oscillating vocal fold models, J Acous Soc Am 132 (2012) 3428-3438.

[27] M. Triep, C. Brucker, W. Schroder, High-speed PIV measurements of the flow downstream of a dynamic mechanical model of the human vocal folds, Exp Fluids 39 (2005) 232-245.

[28] J. Neubauer, Z. Zhang, R. Miraghaie, D. Berry, Coherent structures of the near field flow in a self-oscillating physical model of the vocal folds, J Acous Soc Am 121 (2007) 1102-1118. 
[29] A. Lodermeyer, S. Becker, M. Dollinger, S. Kniesburges, Phase-locked flow field analysis in a synthetic human larynx model, Exp Fluids 56 (2015) $1-13$.

[30] I. Tokuda, R. Shimamura, Effect of level difference between left and right vocal folds on phonation: Physical experiment and theoretical study, J Acous Soc Am 142 (2017) 482-492.

[31] A. Lodermeyer, M. Tautz, S. Becker, M. Dollinger, V. Birk, S. Kniesburges, Aeroacoustic analysis of the human phonation process based on a hybrid acoustic PIV approach, Exp Fluids 59 (2018) 1-15.

[32] I. Titze, S. Schmidt, M. Titze, Phonation threshold pressure in a physical model of the vocal fold mucosa, J Acous Soc Am 97 (1995) 3080-3084.

[33] M. Hirano, S. Kurita, T. Nakashima, Vocal fold physiology: contempory research and clinical issues, College-Hill Press, 1983, Ch. Growth, development and aging of human vocal folds, pp. 22-43.

[34] R. Plant, G. Freed, R. Plant, Direct measurement of onset and offset phonation threshold pressure in normal subjects, J Acous Soc Am 116 (2004) 3640-3646.

[35] M. Mobashir, A. Mohamed, A. Quriba, A. Anany, E. Hassan, Linear measrurements of vocal folds and laryngeal dimensions in freshly excised human larynges, J Voice 32 (2018) 525-529.

[36] C. Vilain, X. Pelorson, C. Fraysse, M. Deverge, A. Hirschberg, J. Willems, Experimental validation of a quasi-steady theory for the flow through the glottis, J. Sound and Vib 276 (2004) 475-490.

[37] F. Alipour, I. Titze, Elastic models of vocal fold tissues, J Acous Soc Am 90 (1991) 1326-1331.

[38] Y. Min, I. Titze, F. Alipour, Stress-strain response of the human vocal ligament, Ann Otol Rhinol Laryngol 104 (1995) 563-569. 
[39] R. Chan, M. Fu, L. Young, N. Tirunagari, Relative contributions of collagen and elastin to elasticity of the vocal fold under tension, Ann Biomed. Eng. 35 (2007) 1471-1483.

[40] S. Smith, S. Thomson, Effect of inferior surface angle on the self-oscillation of a computational vocal fold model, J Acous Soc Am 131 (2012) 40624075 .

[41] R. Scherer, D. Shinwari, K. De Witt, C. Zhang, B. Kucinschi, A. Afjeh, Intraglottal pressure profiles for a symmetric and oblique glottis with a divergence angle of 10 degrees, J Acous Soc Am 109 (2001) 1616-1630.

[42] A. Van Hirtum, X. Pelorson, High-speed imaging to study an autooscillating vocal fold replica for different initial conditions, Int. J. Applied Mech. 9 (1-18).

[43] S. Hwang, M. Litt, W. Forsman, Rheological properties of mucus, Rheologica Acta 8 (1969) 438-448.

[44] W. Schwarz, The rheology of saliva, J Dent Res 66 (1987) 660-664.

[45] S. Lai, Y. Wang, D. Wirtz, J. Hanes, Micro- and macrorheology of mucus, Adv. Drug Deliv. Rev. 61 (2009) 86-100.

[46] J. Phillips, L. Wong, D. Yeates, Bidirectional transepithelial water transport: measurement and governing mechanisms, Biophys J 76 (1999) 869877.

[47] K. Fisher, A. Telser, J. Phillips, D. Yeates, Regulation of vocal fold transepithelial water fluxes, J Appl Physiol 91 (2001) 1401-1411.

[48] M. Sivasankar, K. Fisher, Vocal fold epithelial response to luminal osmotic perturbation, J. Speech Hear Res 50 (2007) 886-898.

[49] A. Miri, Mechanical characterization of vocal fold tissue: a review study, J Voice 28 (6) (2014) 657-666. 